\title{
Factores asociados al índice de reprobación de asignaturas de ciencias básicas del ITSLP
}

María Laura Granja García maria.gg@slp.tecnm.mx

Blanca Estela Ramírez Gámez blanca.rg@slp.tecnm.mx

Angela Rebeca Garcés Rodríguez angela.gr@slp.tecnm.mx

Octavio Villalobos Fernández octavio.vf@slp.tecnm.mx

Irene Zapata Silva irene.zs@slp.tecnm.mx

María Guadalupe Hernández Sierra maria.hs@slp.tecnm.mx

\section{Estudiante: Tanya Jareth Martínez Hernández} L19181091@slp.tecnm.mx

Tecnológico Nacional de México campus Instituto Tecnológico de San Luis Potosí Soledad de Graciano Sánchez, S.L.P., México

\section{RESUMEN}

La finalidad del estudio fue encontrar los factores asociados al índice de reprobación de las asignaturas del Departamento de Ciencias Básicas que afectan a los estudiantes del Instituto Tecnológico de San Luis Potosí, para proponer estrategias que incrementen el índice de aprobación en esta área. Esta investigación tuvo un enfoque cuantitativo, se utilizó la estadística como herramienta básica para el análisis de los datos y sus relaciones, con un alcance descriptivo. Se emplearon los datos del Sistema Integral de Información (SII), para obtener los porcentajes de reprobación de las asignaturas en el periodo de enero a junio 2020. Mediante una encuesta en línea se obtuvieron y analizaron los datos y sus relaciones, en los factores de origen social, personal e institutional en sus 
diferentes dimensiones. De acuerdo a los resultados obtenidos "Los factores de origen social, personal e institucional, se encuentran asociados al índice de reprobación en las asignaturas del Departamento de Ciencias Básicas". De tal manera que siendo una Institución formadora de profesionistas se sugieren estrategias, tales como formar grupos de apoyo para asesorías por parte de los docentes del área de Ciencias Básicas y dar seguimiento a los estudiantes para coadyuvar a bajar el alto índice de reprobación.

Palabras clave: índice de reprobación; educación superior; factores sociales; actores personales; factores institucionales. 


\title{
Factors associated with the failure rate of subjects at the basic sciences department in ITSLP
}

\begin{abstract}
The purpose of the study was to find the factors associated with the failure rate of subjects included in the Basic Science Department that affect students of the "Instituto Tecnológico de San Luis Potosí", to propose strategies that increase the approval rate in this area. This research had a quantitative approach; statistics were used as a basic tool for the analysis of the data and its relationships, with a descriptive scope. Data from the Comprehensive Information System (Sistema Integral de Información, SII) was used to obtain the failure rates of the subjects in the period from January to June 2020. Through an online survey, the data and their relationships were obtained and analyzed, related to social, personal and institutional factors within their different dimensions. According to the results obtained, the hypothesis established in this research is fulfilled as true: "The social, personal and institutional factors are associated with the failure rate in subjects included in the Basic Sciences Department". Being the Instituto Tecnológico de San Luis Potosí a professional training institution, strategies are suggested, such as forming support groups for consultancies from teachers of the Basic Sciences Department in each subject and following up with students to help lower the high failure rate.
\end{abstract}

Keywords: Failure rate, higher education, social factors, personal factors, institutional factors

Artículo recibido: 30 noviembre. 2021 Aceptado para publicación: 29 diciembre 2021 Correspondencia: maria.gg@slp.tecnm.mx Conflictos de Interés: Ninguna que declarar 


\section{INTRODUCCIÓN}

En la actualidad son diversas las dificultades que enfrenta la educación superior en México. El sistema de educación superior mexicano está constituido por más de 4 mil instituciones públicas y privadas que atienden al año alrededor de 4.7 millones de estudiantes (Programa Sectorial derivado del Plan Nacional de Desarrollo (2019-2024). Sin embargo, la mayor parte de los universitarios abandonan sus estudios, cinco de cada diez estudiantes desertan al inicio del segundo año y cuatro de cada diez estudiantes que comienzan el cuarto año, no obtienen el título de licenciatura correspondiente, es decir, no tienen acceso al ámbito productivo del país (Huesca y Castaño, 2007).

De acuerdo con diversos autores (Lara y Valadez, 2011; Álvarez, 2009) la reprobación, la deserción estudiantil y los bajos índices de eficiencia terminal se encuentran entre los problemas más complejos y frecuentes en las instituciones educativas. La problemática que se estudia con mayor frecuencia es la deserción escolar, sin embargo, en la opinión de Álvarez (2009) ésta es precedida por las dificultades que el estudiante va manifestando en forma acumulativa expresadas en la reprobación sistemática de una serie de asignaturas; en consecuencia, opta por abandonar su formación educativa, lo cual repercute de manera negativa en el desarrollo económico, social y cultural de una nación. La reprobación constituye un elemento de un fenómeno más general denominado "fracaso escolar". Dicho fenómeno ha recibido una creciente atención en años recientes, así como la dificultad que presentan algunos alumnos para adquirir los conocimientos, habilidades y actitudes que se enseñan en la escuela (Lozano, 2003), misma que se concreta en el rendimiento escolar, la reprobación y la deserción.

En función de los altos índices de reprobación que prevalecen actualmente en las asignaturas específicas del Departamento de Ciencias Básicas, en las diferentes carreras que se imparten en el Instituto Tecnológico de San Luis Potosí, y de las serias repercusiones que tiene dicho fenómeno a nivel tanto individual como institucional, el propósito de la presente investigación fue analizar la contribución de un conjunto de factores sociales, personales, e institucionales, en la explicación de los índices de reprobación observados en las materias detectadas con los más altos índices de reprobación en dicha institución. Con el fin de proponer estrategias académicas y de orientación educativa que disminuyan los índices de reprobación y bajo desempeño escolar. 
El estudio parte de la premisa de que el proceso de enseñanza aprendizaje en el nivel superior constituye un fenómeno complejo y multivariado. Se plantea que el bajo rendimiento académico de los alumnos puede estar asociado a una amplia gama de factores entre los que podrían señalarse, aspectos relacionados al nivel económico y educativo de la familia, a la configuración del currículum, a la normatividad e infraestructura del proceso educativo e inclusive a características individuales del estudiante, entre otros.

En el Instituto Tecnológico de San Luis Potosí, se imparten carreras de Ingeniería, las cuales incluyen en su retícula asignaturas que corresponden al Departamento de Ciencias Básicas, las cuales son la base de la ingeniería; siendo materias comunes entre las ingenierías: Cálculo diferencial, cálculo integral, cálculo vectorial, ecuaciones diferenciales, álgebra lineal, probabilidad y estadística, y química.

En la Academia de Ciencias Básicas, existe gran preocupación por el aumento progresivo del llamado "fracaso universitario" en sus distintas facetas: abandono, repetición de asignaturas, no presentación de exámenes, ausentismo y pasividad del alumnado. Como lo indica la experiencia, la deserción en muchos casos está concatenada con la repetición reiterada, incluso más allá de esto, son muy pocos los estudiantes que se gradúan en el tiempo estipulado en los planes de estudios.

El modelo académico del instituto contempla diferentes estrategias de apoyo durante la formación del estudiante, que tienen como objetivo incrementar el desarrollo personal y profesional del estudiante, además se cuenta con asesorías y tutorías, sin embargo, pese a los programas y apoyos académicos, en el departamento de Ciencias Básicas, se reportan altos índices de reprobación en varias de sus asignaturas.

Tabla 1.

Asignaturas con altos índices de reprobación.

\begin{tabular}{|l|c|c|c|}
\hline \multicolumn{1}{|c|}{ Asignatura } & $\begin{array}{c}\text { Índice de } \\
\text { reprobación }\end{array}$ & Asignatura & Índice de reprobación \\
\hline Cálculo diferencial & $35 \%$ & Cálculo vectorial & $29 \%$ \\
\hline Cálculo Integral & $46 \%$ & $\begin{array}{c}\text { Ecuaciones } \\
\text { diferenciales }\end{array}$ & $25 \%$ \\
\hline Álgebra lineal & $30 \%$ & Química & $38 \%$ \\
\hline
\end{tabular}

Fuente: Sistema Integral de Información del TecNM/ITSLP 
En la tabla 1 se presenta el listado de las asignaturas que conforman el departamento de Ciencias Básicas, que muestran los índices de reprobación más altos en promedio de todas las carreras en el semestre enero-junio 2021, del Instituto Tecnológico de San Luis Potosí. Se ha visto que, desde hace varios años, se presenta estadísticamente, una tendencia del índice de reprobación muy alto en el Departamento de Ciencias Básicas, por lo anterior, se propone la siguiente investigación: Factores asociados al índice de reprobación en las asignaturas de Ciencias Básicas en los estudiantes del Instituto Tecnológico de San Luis Potosí.

Esta investigación contribuirá a conocer más a fondo a los estudiantes al conocer los factores que inciden en mayor grado al índice de reprobación en el Instituto Tecnológico y servirá de apoyo a los docentes para implementar las estrategias adecuadas para que los estudiantes alcancen un mejor nivel educativo.

\section{ESTRATEGIAS METODOLÓGICAS O MATERIALES Y MÉTODOS}

Para la identificación de los métodos de investigación según refiere (Hernández-Sampieri R., 2018); el estudio realizado es de tipo cuantitativo. Se realizó mediante una encuesta en línea para conocer los factores que se asocian al alto índice de reprobación de las materias de Ciencias Básicas en los estudiantes. Para la recolección de los datos y posterior análisis de los mismos, se utilizó la estadística descriptiva.

\section{Método y organización del trabajo de campo.}

\section{Se llevaron a cabo las siguientes acciones:}

a) Se utilizó Microsoft Forms de Google para la elaboración del cuestionario. La encuesta se diseñó con tres tipos de respuestas:

- De varias opciones

- De solo una opción

- Escala de Likert

- Abierta

b) Las respuestas de la encuesta se obtuvieron mediante la difusión por medios electrónicos entre los estudiantes del Tecnológico de San Luis Potosí, a través de un link para ingresar al cuestionario. Se obtuvieron 593 respuestas de todas las carreras que se imparten en el instituto.

c) Recolección y tratamiento de los datos. 


\section{RESULTADOS Y DISCUSIÓN}

Como desenlace del estudio, se identificaron los principales factores asociados al índice de reprobación, la interpretación de los resultados se hizo por medio de un análisis de estadística descriptiva e inferencial, ya que se pretende describir el objeto de estudio e inferir las causas que provocan el índice de reprobación en las asignaturas de Ciencias Básicas.

Lo anterior, de acuerdo a las respuestas obtenidas en la encuesta realizada para este propósito y sistematizando la información con base a los factores de origen social, personal e institucional con sus diferentes dimensiones, donde participaron 593 estudiantes inscritos en el Instituto Tecnológico de San Luis Potosí, quienes cursan del segundo semestre en adelante.

\subsection{Factores de origen social}

3.1.1 Dimensión sociodemográfica. Los factores de origen social se desglosan en dimensión sociodemográfica y dimensión diferencias sociales. Respecto a la dimensión sociodemográfica (Tabla 2), se obtuvieron los siguientes datos: referente al género de los estudiantes, mujeres (305), hombres (286) y estudiantes que prefirieron no decir su género (2) (Gráfica 1); edades de los estudiantes de 17 años (3), de 18 años (32), de 19 años (140) y de más de 20 años (418) estudiantes (Gráfica 2); en lo que se refiere al estado civil de dichos estudiantes: solteros (556), casados (12) y en unión libre (25) (Gráfica 3).

Por lo anterior, se concreta, en cuanto al género de los estudiantes, que hay un porcentaje un poco mayor de mujeres, y que los rangos de edades de los estudiantes se perfilan de más de 20 años, y que el 94\% permanecen solteros.

\section{Factores de origen social}

Tabla 2.

Factores de origen Social; dimensión sociodemográfica.

DIMENSIÓN: SOCIO DEMOGRÁFICA
\begin{tabular}{|c|c|c|c|c|c|c|c|c|c|}
\hline \multicolumn{2}{|l|}{ SEXO } & \multicolumn{2}{|c|}{ EDAD } & \multicolumn{3}{|c|}{ ESTADO CIVIL } \\
\hline Mujer & Hombre & $\begin{array}{l}\text { Prefiere } \\
\text { no decir }\end{array}$ & $\mathbf{1 7}$ años & $\mathbf{1 8}$ años & $\mathbf{1 9}$ años & $\begin{array}{l}\text { Más de } \\
\mathbf{2 0} \text { años }\end{array}$ & Casado & Soltero & $\begin{array}{l}\text { Unión } \\
\text { libre }\end{array}$ \\
\hline 305 & 286 & 2 & 3 & 32 & 140 & 418 & 12 & 556 & 25 \\
\hline $52 \%$ & $48 \%$ & $0 \%$ & $1 \%$ & $5 \%$ & $24 \%$ & $70 \%$ & $2 \%$ & $94 \%$ & $4 \%$ \\
\hline
\end{tabular}

Fuente: Elaboración propia 
Gráfica 1. Sexo de los estudiantes.

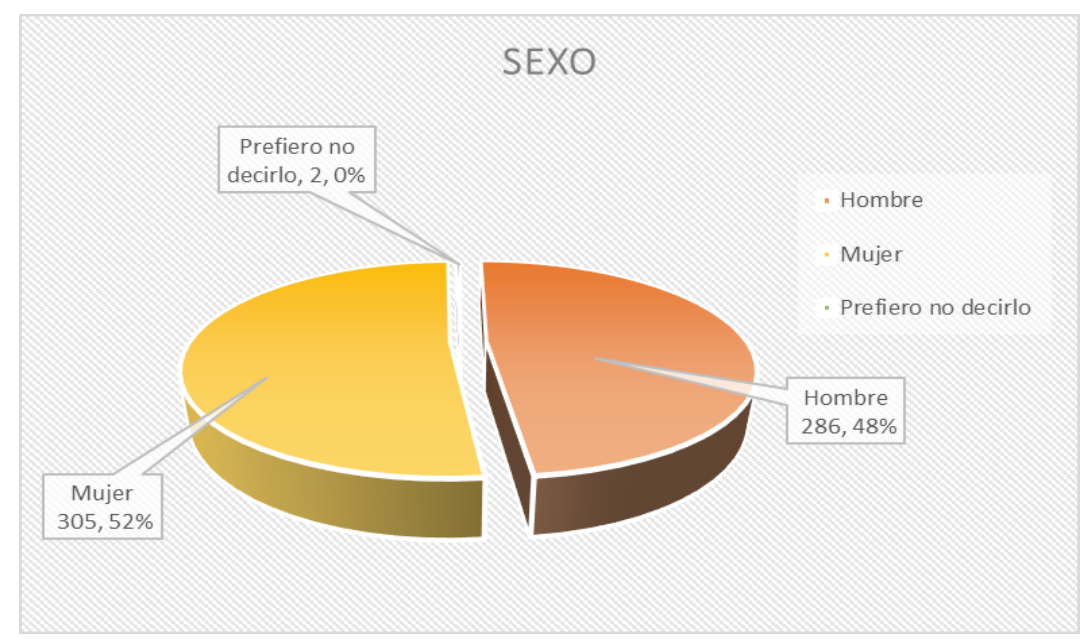

Fuente: Elaboración propia

Gráfica 2. Edad de los estudiantes.

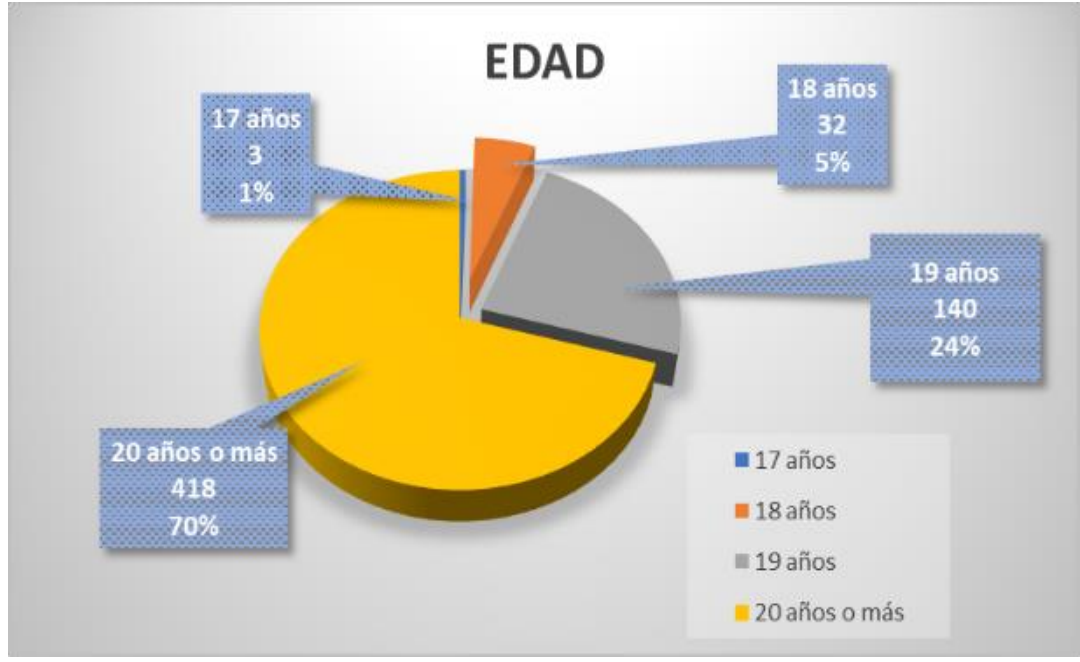

Fuente: Elaboración propia

Gráfica 3. Estado civil de los estudiantes.

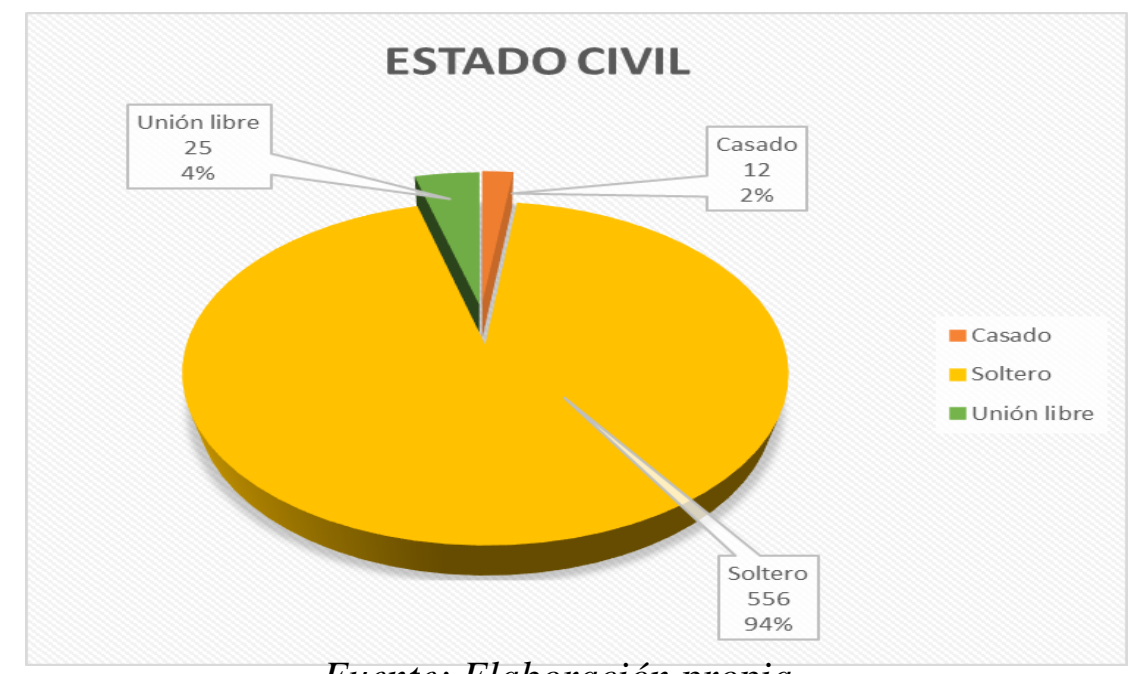

Fuente: Elaboración propia 
3.1.2 Dimensión diferencias sociales. Los resultados arrojados de la encuesta de esta dimensión (Tabla 3), denotan el gran número de estudiantes que trabajan y estudian al mismo tiempo (333) y los que solo estudian (260) (Gráfica 4).

Respecto al rubro de su manutención, se encuentra que el padre es quien por lo general sostiene los estudios de los alumnos (333), la madre (143) y otros medios (112); además se encontró que, los ingresos mayoritariamente se encuentran en el rango de menos de seis mil pesos mensuales (281), de seis mil a doce mil (217).

En lo que se refiere al nivel académico de los padres se puede ver que el mayor número se encuentra en el nivel de secundaria (215) para la mamá y (112) para el papá, y del nivel de preparatoria con (176) de mamá y (174) para papá, y con un porcentaje mucho más bajo para el nivel de licenciatura (95) que cursó mamá y (88) para el nivel académico del papá, es decir, prevalecen el nivel de secundaria y preparatoria.

En cuanto a la escuela de procedencia de los estudiantes se puede determinar que provienen en mayor cantidad del Colegio de Bachilleres (167) Centro de Bachillerato de Estudios Tecnológicos y de Servicios (141), y Colegio de Estudios Científicos y Tecnológicos (82) (Gráfica 5); en lo que se refiere a la obtención de apoyo de algún tipo de beca (238)respondieron que han recibido algún beneficio y (355) no lo han tenido.

Referente al bachillerato cursado la mayoría de los alumnos refieren haber llevado una capacitación diferente (232), físico matemático (205), socio administrativo (104) y químico biológico (31) (Gráfica 6).

Tabla 3. Factores de origen Social; diferencias sociales.

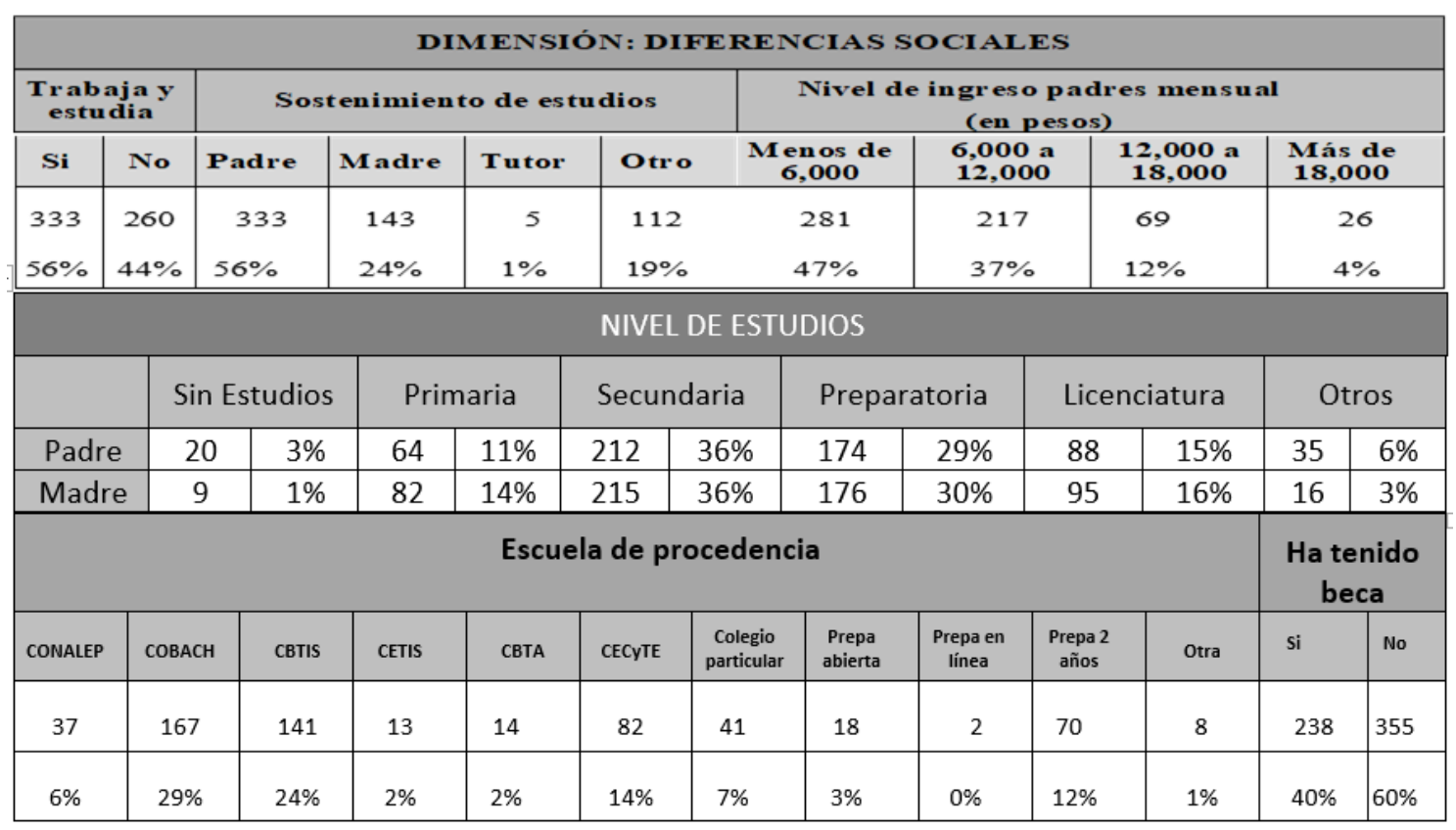


Gráfica 4. Estudiantes que trabajan y estudian.

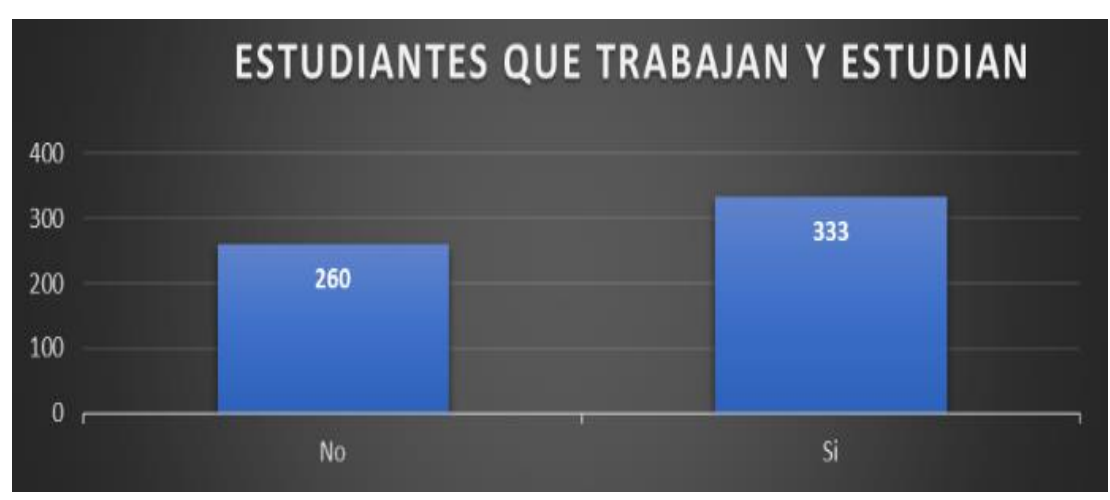

Fuente: Elaboración propia

Gráfica 5. Escuela de procedencia de los estudiantes

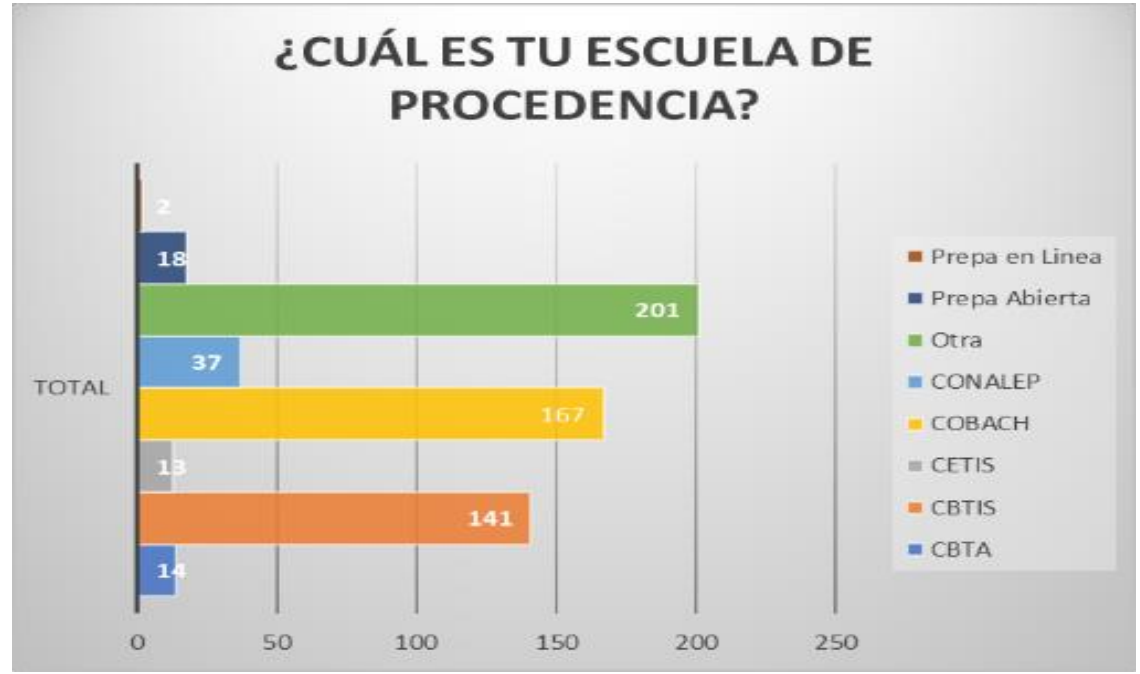

Fuente: Elaboración propia

Gráfica 6. Bachilleratos cursados por los estudiantes.

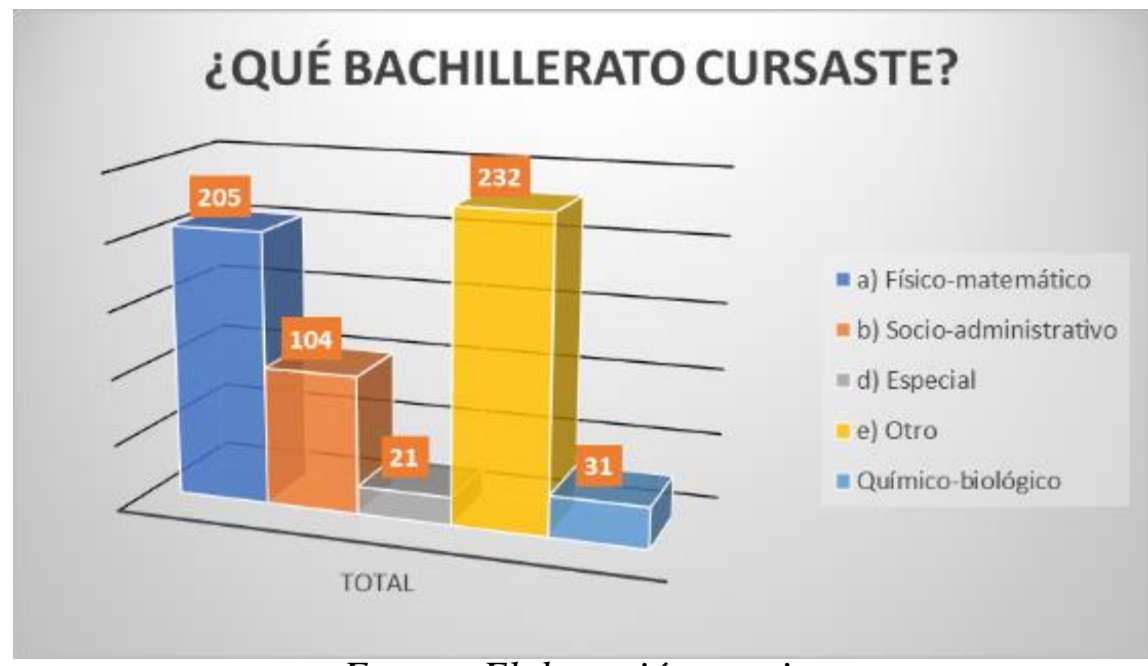

Fuente: Elaboración propia 


\subsection{Factores de origen personal}

3.2.1 Dimensión recursos académicos. Los factores de origen personal, se dividen en dimensión recursos académicos y dimensión desempeño académico. Por lo que se refiere a la dimensión recursos académicos, se encontró que únicamente (283) estudiantes sí recibieron orientación vocacional en el nivel medio superior, contra (310) que no tuvieron dicha orientación (Gráfica 7); por lo que (264) alumnos opinan que les hizo falta orientación y (329) creen haber recibido la orientación necesaria (Gráfica 8); además se encontró que (444) afirman haber conocido el plan de estudios de la carrera que eligieron, mientras que (149) alumnos dicen no haber visto el plan anteriormente (Gráfica 9).

Gráfica 7. Estudiantes que recibieron orientación vocacional en el nivel medio superior.

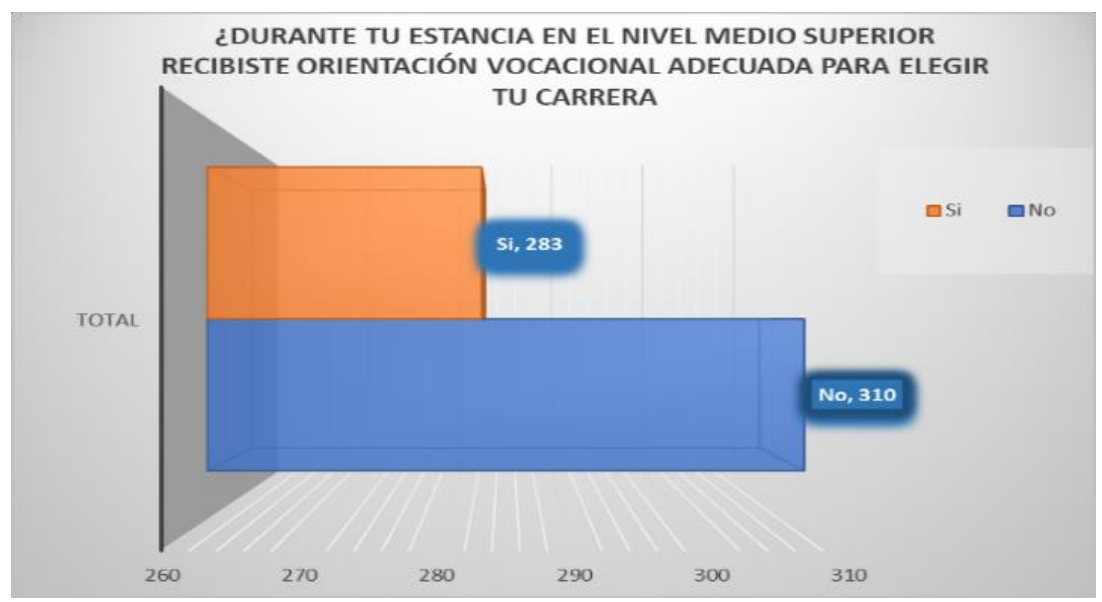

Fuente: Elaboración propia

Gráfica 8. Estudiantes que no recibieron orientación vocacional antes de ingresar al Instituto

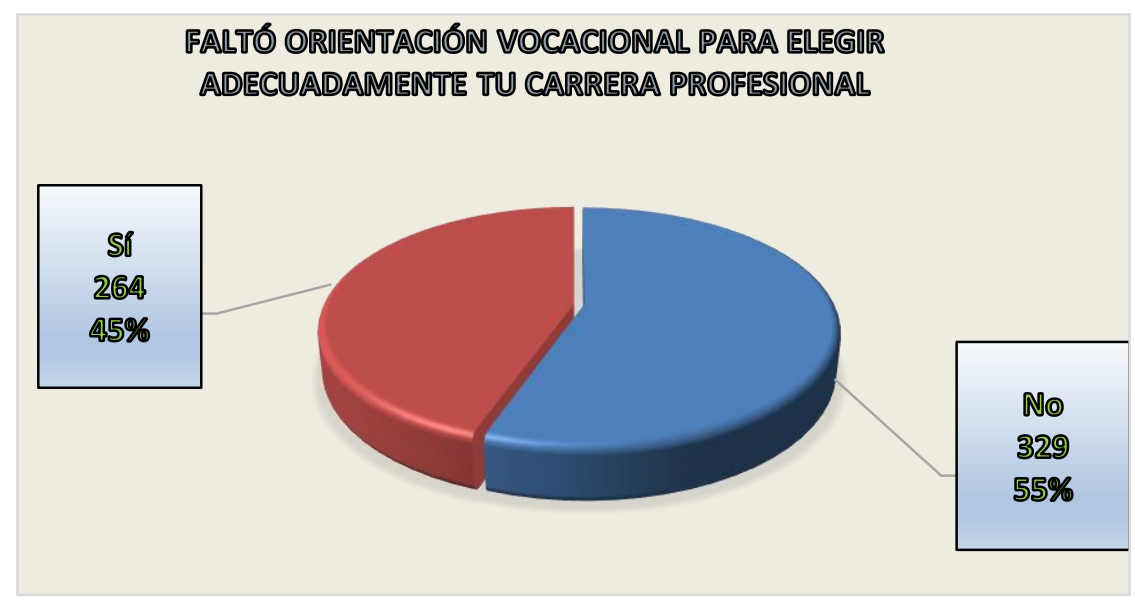

Fuente: Elaboración propia 
Gráfica 9. Estudiantes que no recibieron orientación vocacional antes de ingresar al Instituto.

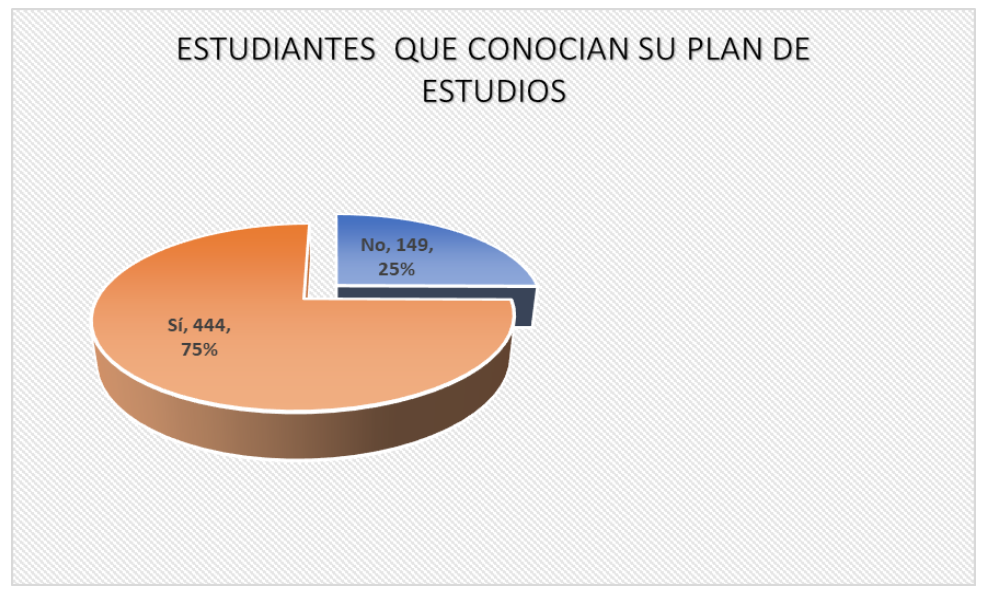

Fuente: Elaboración propia

3.2.2 Dimensión desempeño académico. En esta encuesta participaron estudiantes de cada una de las carreras que se imparten en el Instituto Tecnológico de San Luis Potosí, la muestra quedó conformada de la siguiente manera: Ingeniería Mecatrónica (155), Ingeniería Industrial (108), Ingeniería en Sistemas Computacionales (27), Ingeniería Informática (15), Ingeniería en Gestión Empresarial (78), Ingeniería Electrónica (18), Ingeniería Eléctrica (16), Ingeniería Mecánica (38), Licenciatura en Administración (82), y Licenciatura en Turismo (55) (Gráfica 10).

Gráfica 10. Carreras que estudian los participantes de esta investigación.

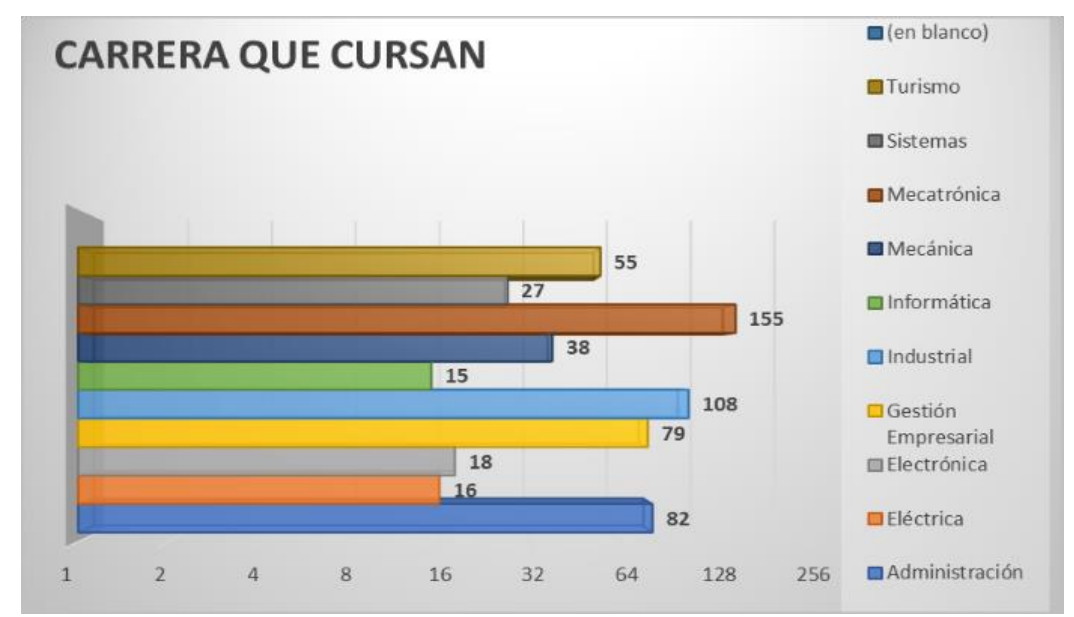

Fuente: Elaboración propia 
Dichos estudiantes se encuentran en diferentes etapas de su carrera profesional, sin embargo, la muestra requirió alumnos que hayan concluido su primer semestre, por lo que la muestra se conformó por (122) segundo semestre, (112) tercer semestre, (74) cuarto semestre, (76) quinto semestre, (46) sexto semestre, (64) séptimo semestre, (26) octavo semestre, (42) noveno, y (31) de más de nueve semestres (Gráfica 11).

Gráfica 11. Semestre que cursan los participantes de esta investigación

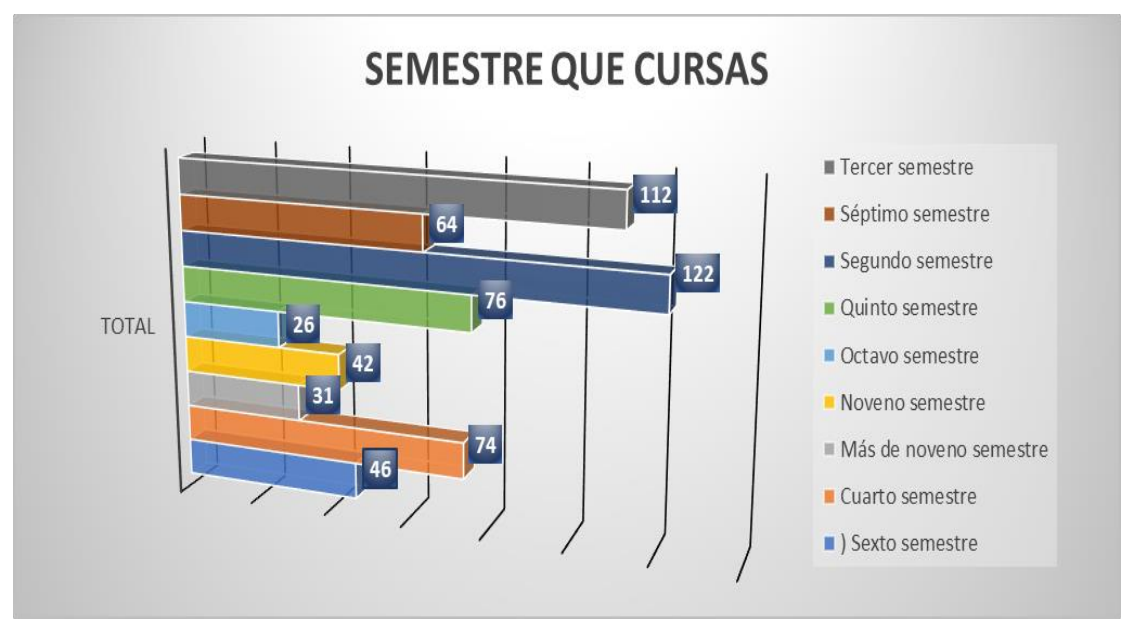

.Fuente: Elaboración propia

Respecto al número de materias reprobadas en Ciencias Básicas, se encontró que (430) estudiantes han reprobado una materia, (88) dos materias, (33) tres materias, (23) cuatro materias, y (19) más de cuatro materias (Gráfica 12). En este tenor, (500) estudiantes refieren que, a veces se les dificulta comprender las clases, a (47) nunca se les dificulta, mientras que a (19) casi siempre y (27) siempre se les dificulta (Gráfica 13).

Gráfica 12. Materias reprobadas de los participantes de esta investigación.

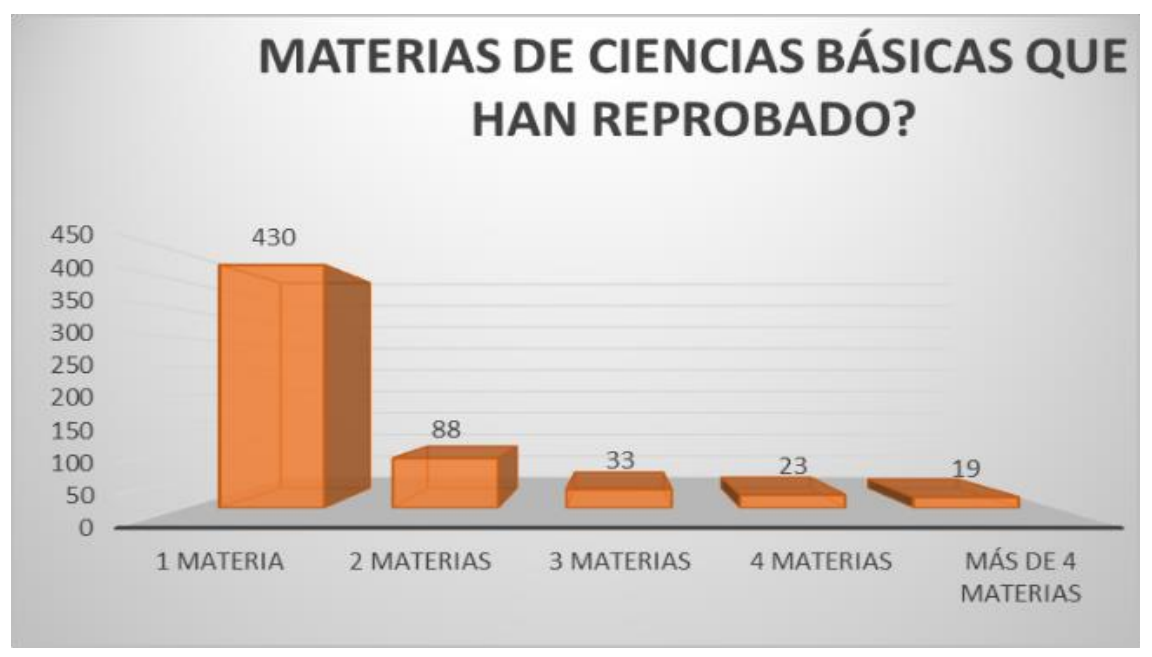

Fuente: Elaboración propia 
Gráfica 13. Dificultades para comprender las clases

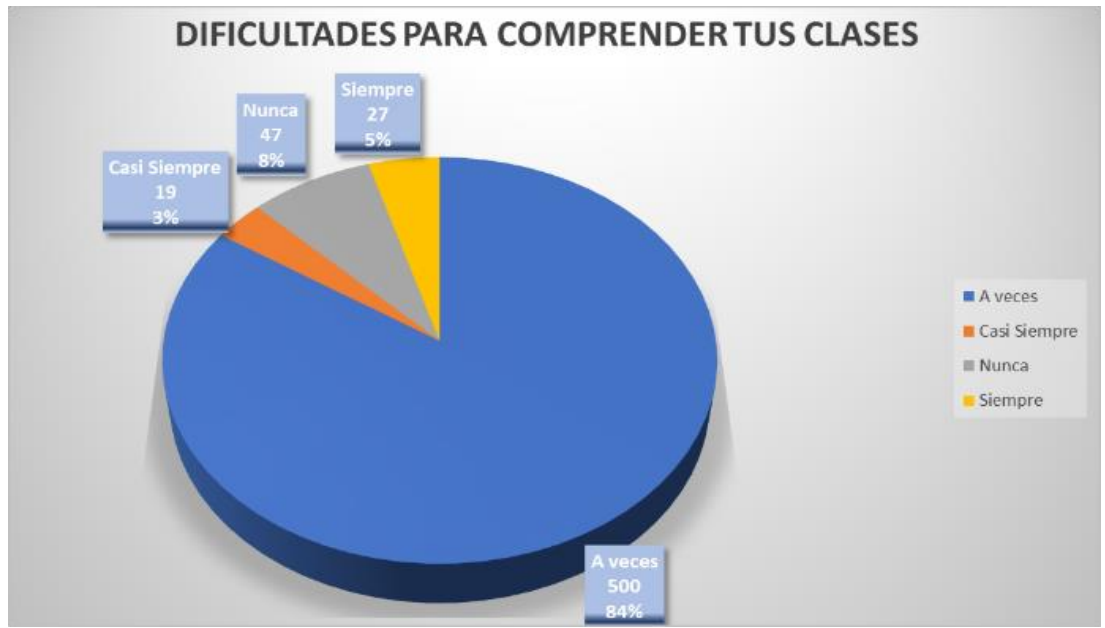

Fuente: Elaboración propia

En esta línea, el tiempo de estudio que los estudiantes refieren en la encuesta refleja que (321) dedican de dos a cuatro horas para estudiar y hacer tareas, (167) más de cuatro horas y (104) menos de dos horas (Gráfica 14).

Gráfica 14. Tiempo dedicado diariamente para hacer tareas.

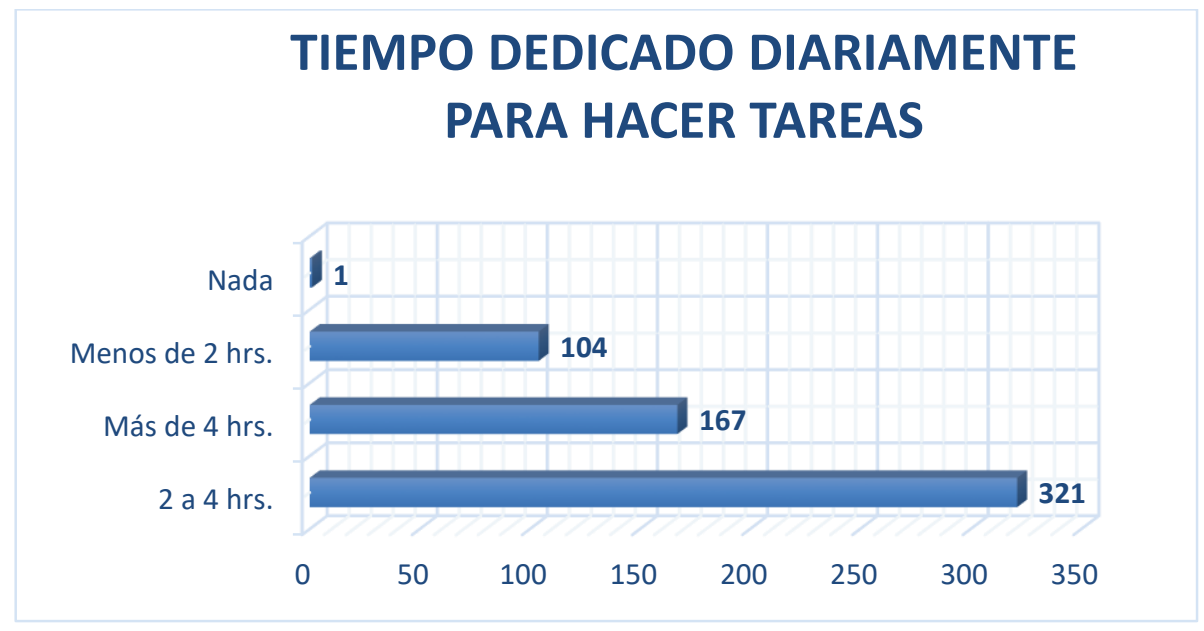

Fuente: Elaboración propia

En este rubro, se encontró que, dentro de las técnicas empleadas por los estudiantes, leer apuntes de clase diariamente es la técnica más utilizada (390), seguida por elaboración de resúmenes de clase (129), creación de síntesis y esquemas (79) y la menos utilizada es leer libros diariamente (42) (Gráfica 15). 
Gráfica 15. Técnicas de estudias utilizada con frecuencia

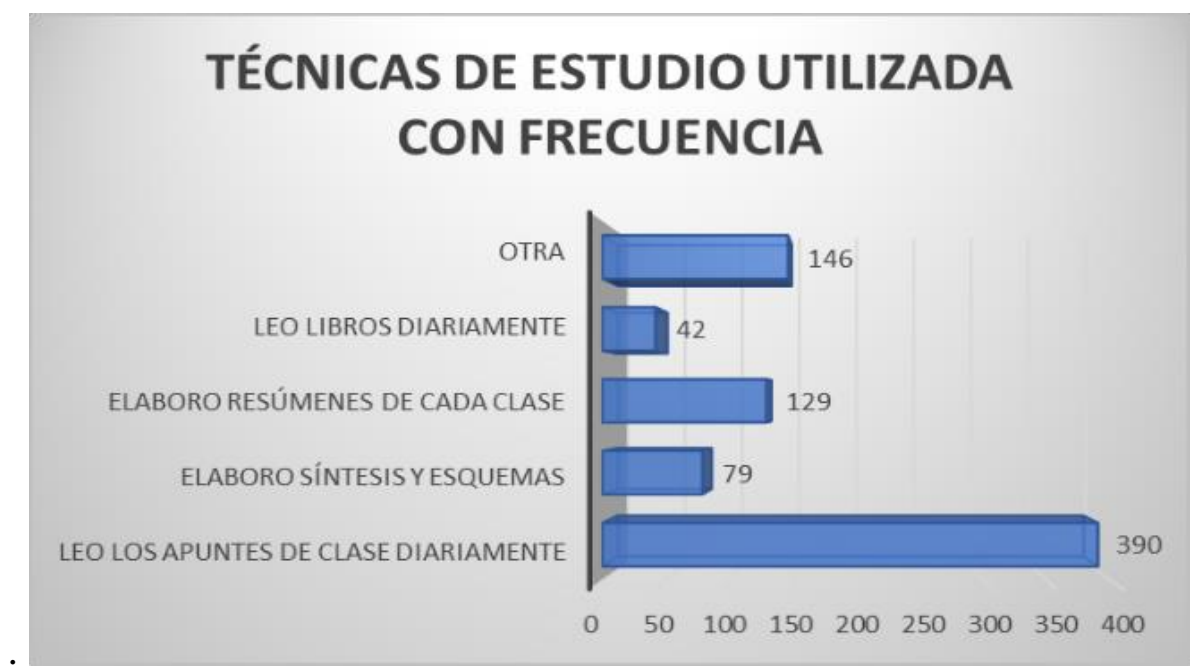

Fuente: Elaboración propia

Respecto a la preparación para sus exámenes, se encontró que en su mayoría (285) estudia un día previo al examen, (151) estudian una vez a la semana, (124) cada tercer día, y únicamente (91) estudian todos los días, mientras que, (46) simplemente no estudian (Gráfica 16).

Gráfica 16. Preparación para presentar exámenes.

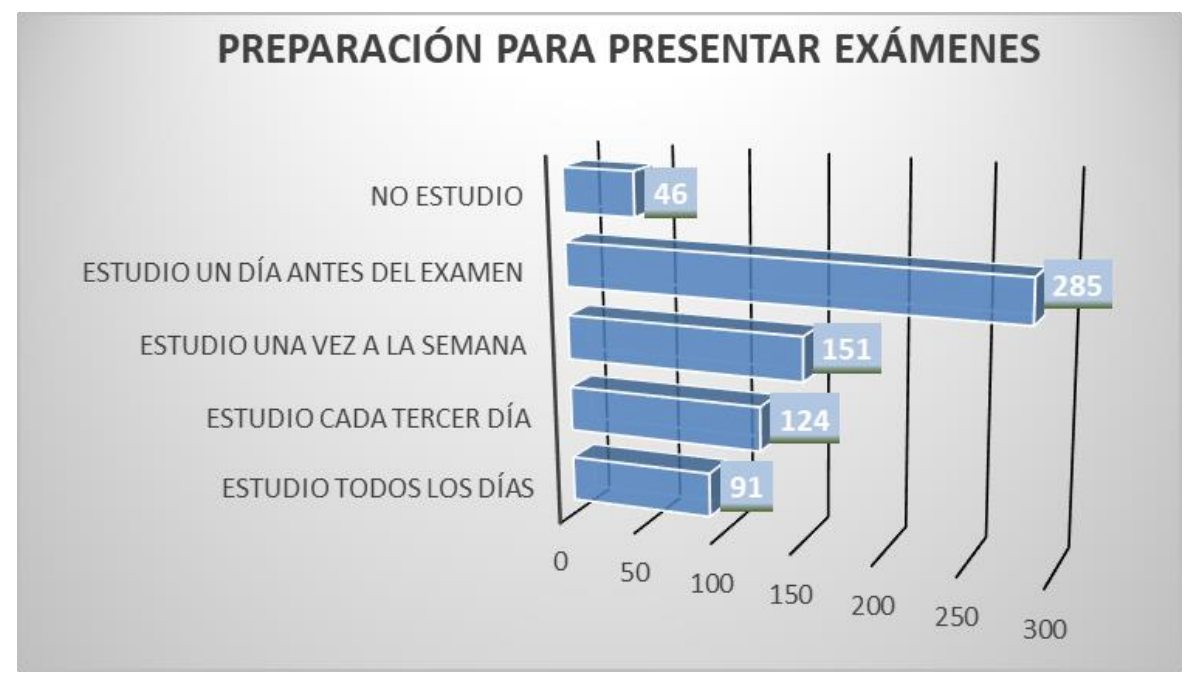

Fuente: Elaboración propia

Esta situación refleja el alto índice de reprobación en las materias de Ciencias Básicas, siendo Cálculo Integral la materia que presenta el número más alto de reprobados (161), seguida por Cálculo Diferencial (155), Probabilidad y Estadística (72) y Química (69) (Gráfica 17). 
Gráfica 17. Índice de reprobación en las materias de Ciencias Básicas

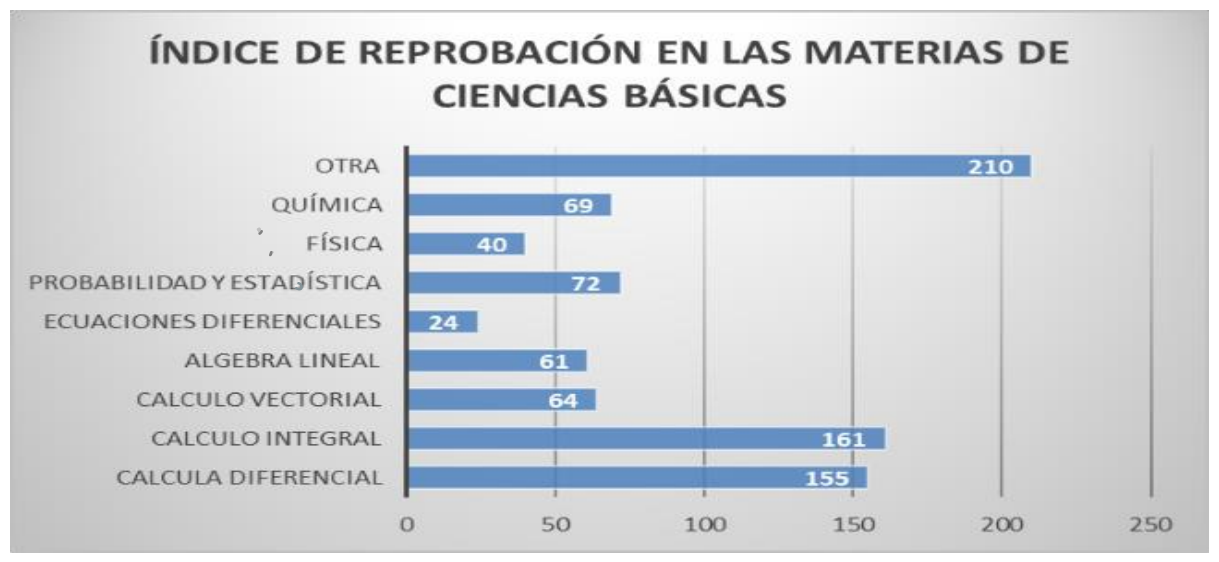

Fuente: Elaboración propia

\subsection{Factores de origen institucional.}

3.3.1 Dimensión infraestructura. En este rubro lo estudiantes comentaron que las instalaciones que ofrece el Instituto Tecnológico de San Luis Potosí se encuentran en muy buenas condiciones, por lo que se infiere que, los salones, laboratorios, auditorios, áreas deportivas, servicios sanitarios, oficinas, áreas verdes son del agrado de los estudiantes, lo que conlleva a percibir con satisfacción el ambiente escolar, así como una alta apreciación en el servicio que se recibe por parte del personal administrativo, sin embargo, en cuanto a los docentes, se tiene una impresión regular acerca de su desempeño (Tabla 4).

Tabla 4 Factores de origen institucional; infraestructura y recursos docentes.

\begin{tabular}{|c|c|c|c|c|c|c|c|c|c|c|c|}
\hline \multicolumn{12}{|c|}{ FACTORES DE ORIGEN INSTITUCIONAL } \\
\hline \multicolumn{12}{|c|}{ DIMENSIÓN: Infraestructura } \\
\hline \multicolumn{12}{|c|}{ Características ITSLP } \\
\hline \multicolumn{3}{|c|}{ Instalaciones } & \multicolumn{3}{|c|}{ Ambiente } & \multicolumn{4}{|c|}{ Profesores } & \multicolumn{2}{|c|}{ Servicio } \\
\hline \multicolumn{3}{|c|}{ Muy bien } & \multicolumn{3}{|c|}{ Muy bien } & \multicolumn{4}{|c|}{ Regular } & \multicolumn{2}{|c|}{ Muy bien } \\
\hline \multicolumn{3}{|c|}{$82 \%$} & \multicolumn{3}{|c|}{$78 \%$} & \multicolumn{3}{|c|}{$58 \%$} & & \multicolumn{2}{|l|}{$71 \%$} \\
\hline \multicolumn{12}{|c|}{ DIMENSIÓN: Recursos Docentes } \\
\hline \multicolumn{5}{|c|}{ Cómo consideran a los docentes } & \multicolumn{7}{|c|}{ Práctica docente } \\
\hline \multicolumn{2}{|c|}{$\begin{array}{l}\text { Comprensivos } \\
\text { y tolerantes }\end{array}$} & \multicolumn{2}{|c|}{$\begin{array}{l}\text { Arbitrarios, } \\
\text { déspotas y } \\
\text { prepotentes }\end{array}$} & Otra forma & \multicolumn{2}{|c|}{ Excelente } & \multicolumn{2}{|c|}{$\begin{array}{c}\text { Muy } \\
\text { buena }\end{array}$} & Buena & Regular & Mala \\
\hline 439 & & \multicolumn{2}{|l|}{62} & 92 & \multicolumn{2}{|c|}{77} & \multicolumn{2}{|c|}{198} & 232 & 75 & 11 \\
\hline $74 \%$ & \multicolumn{3}{|c|}{$10 \%$} & $16 \%$ & \multicolumn{2}{|c|}{$13 \%$} & \multicolumn{2}{|c|}{$33 \%$} & $39 \%$ & $13 \%$ & $2 \%$ \\
\hline \multicolumn{3}{|c|}{ Sistema de enseñanza } & & lanificació & in de cla & & & Cum & $\begin{array}{r}\text { plimient } \\
\text { plan }\end{array}$ & $\begin{array}{l}\text { o de activid } \\
\text { leadas }\end{array}$ & des \\
\hline Tradicional & & npetencias & Siempre & $\begin{array}{c}\text { Casi } \\
\text { siempre }\end{array}$ & A veces & Nur & nea & Siempre & $\begin{array}{c}\text { Casi } \\
\text { siempre }\end{array}$ & A veces & Nunca \\
\hline 367 & & 226 & 181 & 257 & 143 & 13 & 2 & 169 & 125 & 293 & 6 \\
\hline $62 \%$ & & $38 \%$ & $30 \%$ & $43 \%$ & $25 \%$ & $2 c$ & $\%$ & $28 \%$ & $21 \%$ & $50 \%$ & $1 \%$ \\
\hline $\mathbf{E x}$ & me & nes aplica & dos por & materia & & & & & Evaluacio & ón & \\
\hline Uno & & Dos & Tres & Más c & de tres & & $\begin{array}{l}\text { amer } \\
\text { crito }\end{array}$ & $\begin{array}{r}\text { Exá } \\
\mathbf{t}\end{array}$ & $\begin{array}{l}\text { menes y } \\
\text { areas }\end{array}$ & Proyectos & Otros \\
\hline 106 & & 21 & 51 & & 15 & & 24 & & 538 & 8 & 23 \\
\hline $18 \%$ & & $4 \%$ & $8 \%$ & & $0 \%$ & & $4 \%$ & & $91 \%$ & $1 \%$ & $4 \%$ \\
\hline
\end{tabular}


3.3.2 Dimensión recursos docentes. En relación a la percepción que tienen los estudiantes sobre los docentes, (439) los consideran comprensivos y tolerantes, y por otro lado (62) como arbitrarios, déspotas y prepotentes (Gráfica 18). En cuanto a la práctica docente (198) estudiantes la consideran buena, (232) muy buena, (75) regular y solamente (11) la consideran mala práctica (Gráfica 19).

Gráfica 18. Cómo perciben los estudiantes a sus maestros

\section{CÓMO PERCIBEN LOS ESTUDIANTES A SUS MAESTROS}

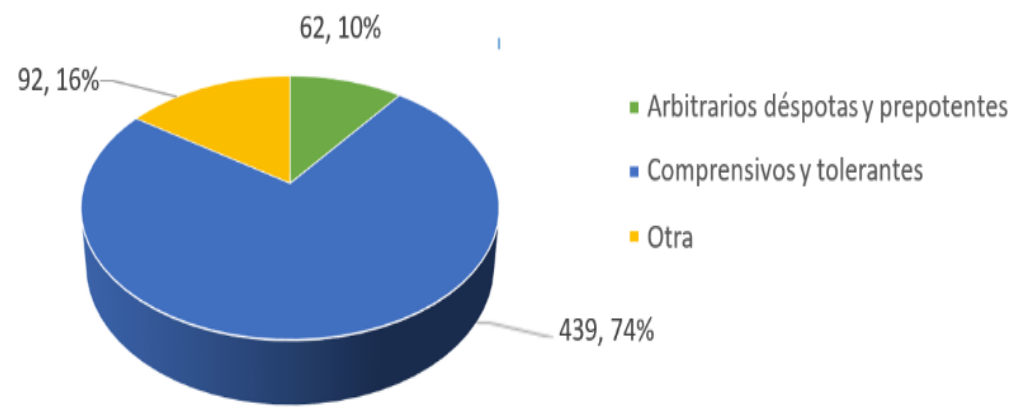

Fuente: Elaboración propia

Gráfica 19. Cómo perciben los estudiantes la práctica docente de sus maestros.

\section{PRÁCTICA DOCENTE DE LOS MAESTROS}

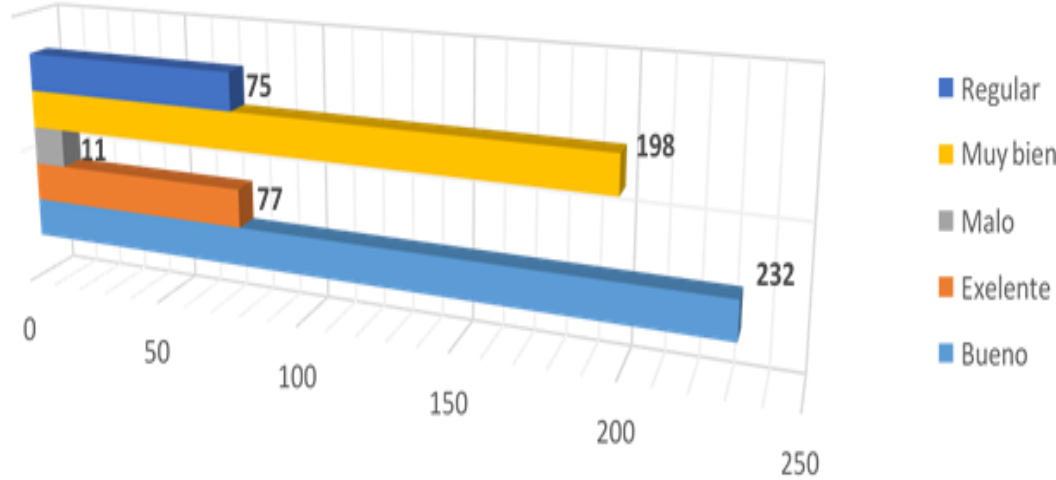

Fuente: Elaboración propia

De acuerdo a los estudiantes, el sistema de enseñanza más utilizado por sus docentes es de forma tradicional (367) y (226) por competencias (Gráfica 20). 
Gráfica 20. Sistema de enseñanza-aprendizaje usado por los maestros.

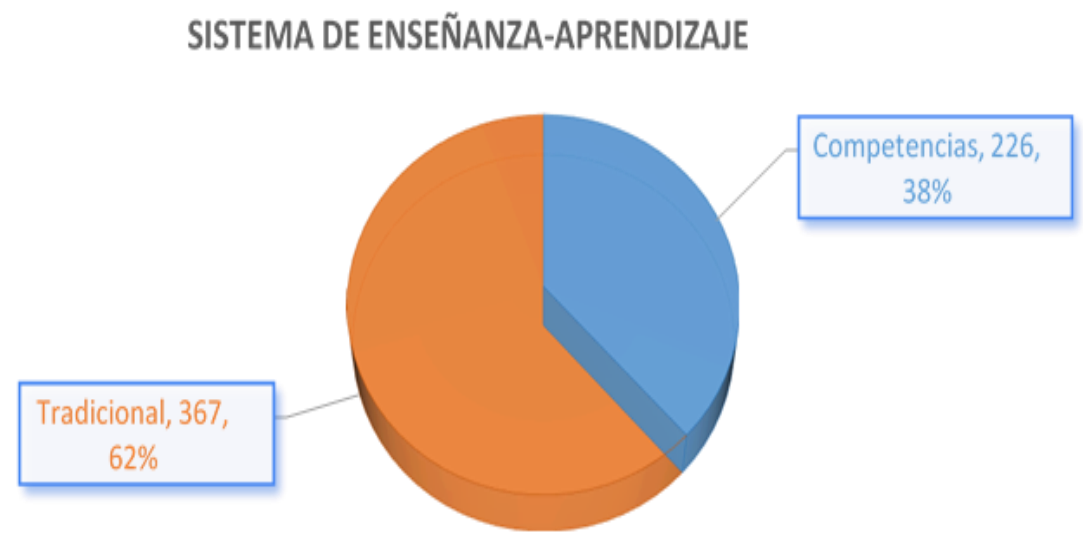

Fuente: Elaboración propia

Opinan que sus docentes siempre elaboran una planificación de clases (181), casi siempre la elaboran (257), a veces la realizan (143) y nunca la hacen solo (12) (Gráfica 21).

Gráfica 21. Sistema de enseñanza-aprendizaje usado por los maestros.

\section{MAESTROS PLANIFICAN SUS CLASES DURANTE EL SEMESTRE}

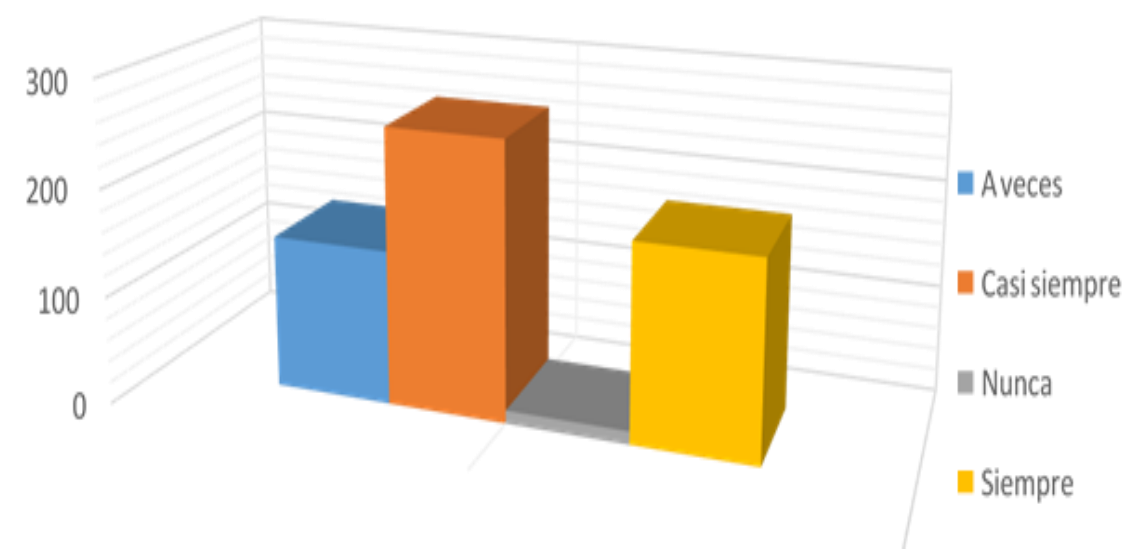

Fuente: Elaboración propia

Respecto al cumplimiento de las actividades planeadas para las clases, la mayoría indica que sus docentes a veces lo llevan a cabo (293) (Gráfica 22). 
Gráfica 22. Percepción de los alumnos respecto al cumplimiento y seguimiento de las planeaciones de clases por parte de los maestros

\section{LOS MAESTROS CUMPLEN CON SUS PLANEACIONES}

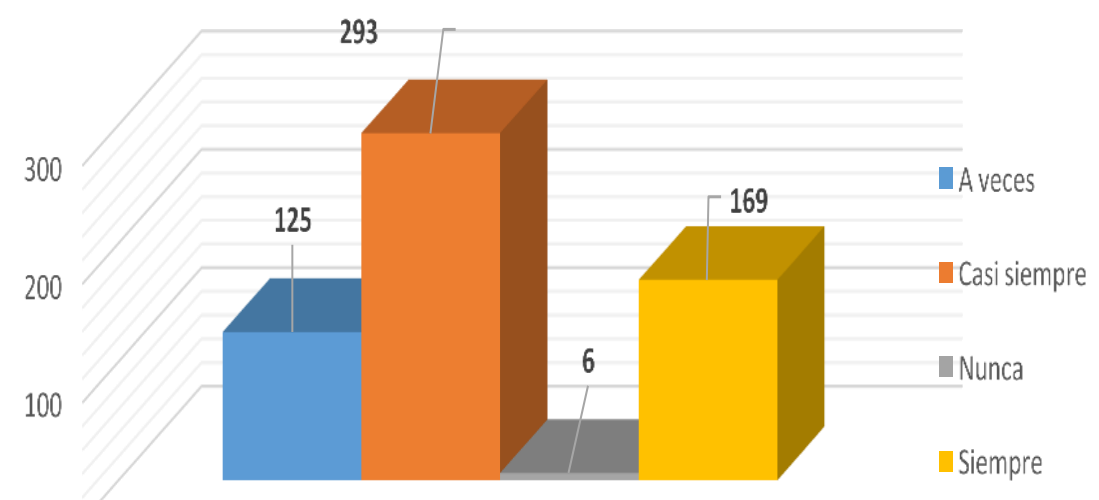

.Fuente: Elaboración propia

Durante el semestre, los alumnos dicen que sus docentes aplican más de tres exámenes parciales (415), tres exámenes (51), dos exámenes (21) y solo un examen (106) (Gráfica 23).

Gráfica 23. Número de exámenes parciales por semestre.

\section{NÚMERO DE EXÁMENES PARCIALES}

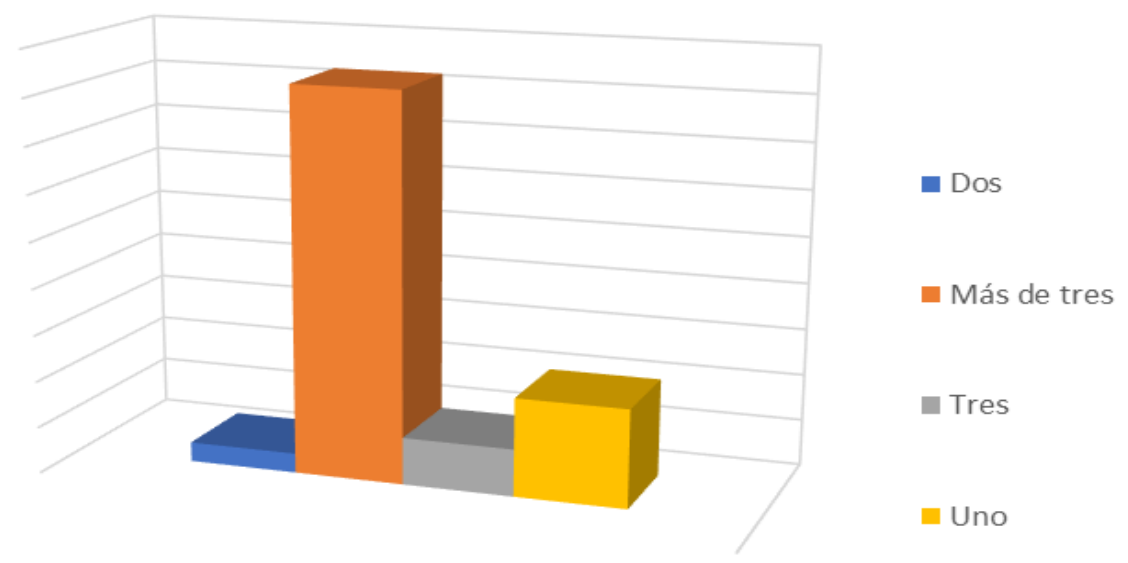

Fuente: Elaboración propia

Siendo el tipo de evaluación mayormente utilizada por sus docentes los exámenes y tareas (538) y el menor por proyectos (8) (Gráfica 24). 
Gráfica 24. Tipo de evaluaciones empleadas por los docentes.

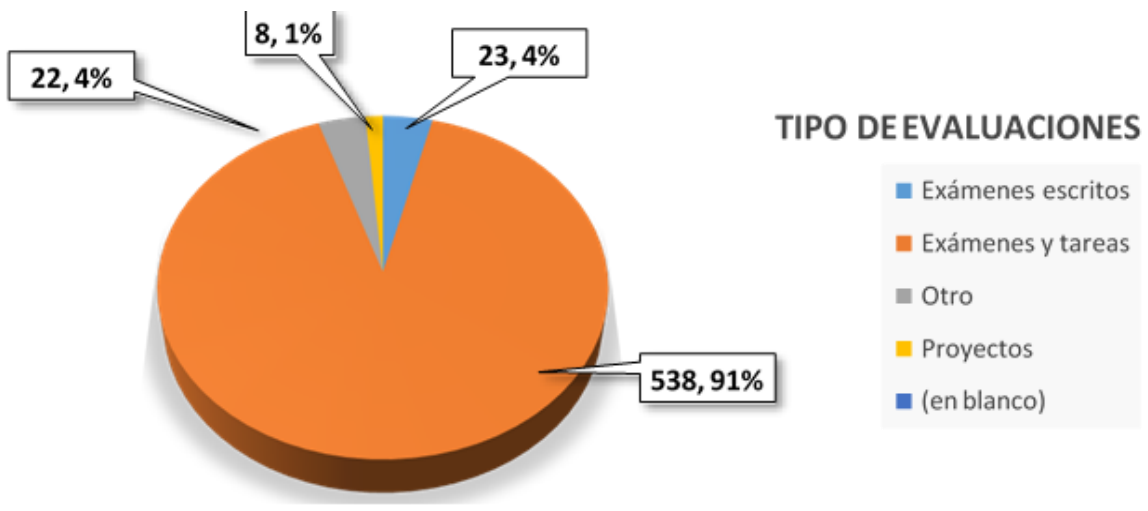

Fuente: Elaboración propia

\subsubsection{Hallazgos}

Es preciso contrastar los resultados discutidos previamente, para visualizar el alcance de esta investigación, por lo que se requiere establecer una relación entre los diferentes factores y las dimensiones que los conforman, y de esta forma esclarecer la pregunta de investigación:

¿Cómo influyen los factores asociados al índice de reprobación en las asignaturas de Ciencias Básicas en los estudiantes del Instituto Tecnológico de San Luis Potosí?

Por lo anterior, se identificaron y sistematizaron los resultados obtenidos en los diferentes factores para tratar de responder las preguntas subordinas de esta investigación. Respecto a la primera pregunta subordinada, ¿Cuáles son los factores de origen social de mayor incidencia en el índice de reprobación en Ciencias Básicas en los estudiantes del Instituto Tecnológico de San Luis Potosí?

Al referirnos al aspecto social, Bourdieu declara que la sociedad opera como un espacio social caracterizado por posiciones distintas y coexistentes, definidas por relaciones de proximidad o distanciamiento y relaciones de orden jerárquico, de acuerdo a dos principios de diferenciación: el capital económico y el capital cultural (Bourdieu y Passeron, 1973, en Ávila Francés, 2005).

Con relación a la clase social, las investigaciones al respecto informan que, a medida que se asciende en la escala social, los resultados y expectativas futuras son mejores. De acuerdo a Lozano (2003) los alumnos de clase alta presentan un mejor uso de estrategias metacognitivas que los de clase social más baja. La influencia de la clase social está mediada por el nivel cultural que, a su vez, determina las expectativas, valores y actitudes de la familia respecto a la educación (Gráfica 25). 
Gráfica 25. Tipo de evaluaciones empleadas por los docentes.

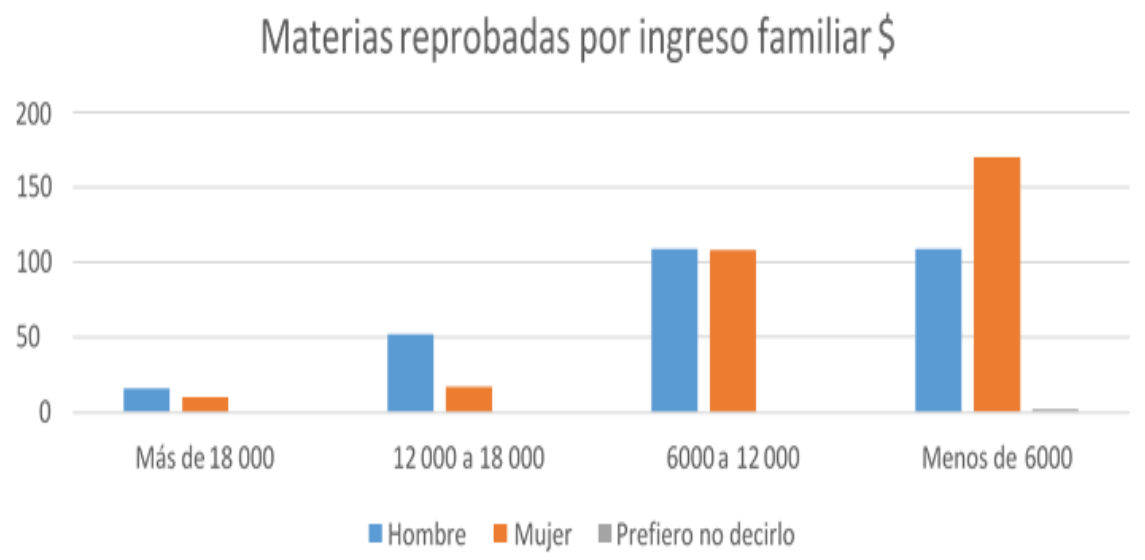

Fuente: Elaboración propia

Por lo anterior se encontró que, en el factor de origen social, los aspectos de mayor relevancia se encontraron en materias reprobadas debido a situaciones de estudiar y trabajar (Gráfica 26).

Gráfica 26. Relación de materias reprobadas respecto a estudiantes que estudian y trabajan.

\section{Materias reprobadas por estudiantes que trabajan}

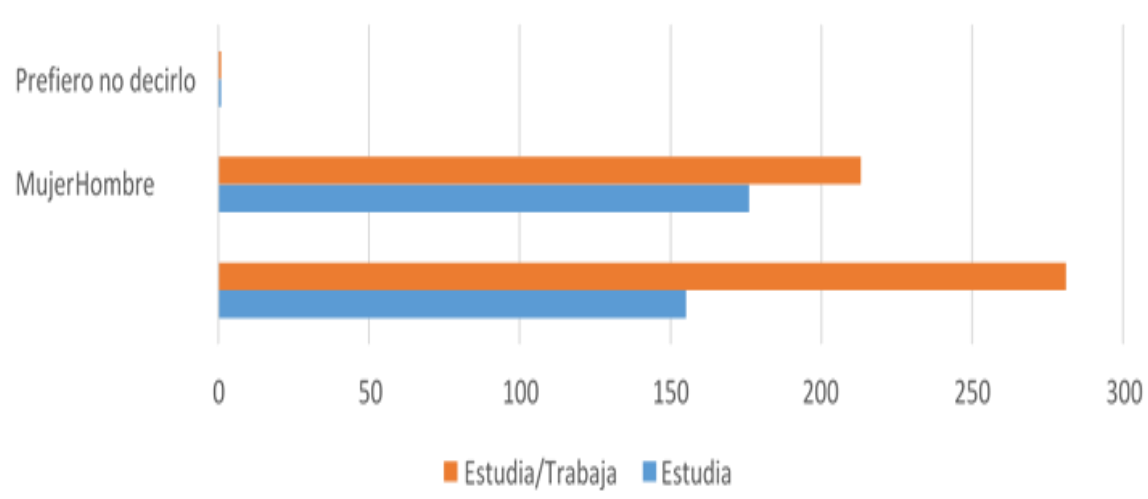

Fuente: Elaboración propia

En cuanto a la segunda pregunta subordinada, ¿Cuáles son los factores de origen personal de mayor incidencia en el índice de reprobación en Ciencias Básicas en los estudiantes del nstituto Tecnológico de San Luis Potosí?, Lozano (2003) establece que entre las variables personales más frecuentes se encuentran la motivación y el autoconcepto (Gráfica 27). 
Gráfica 27. Materias reprobadas por carrera.

\section{Materias reprobadas por carrera (autoconcepto)}

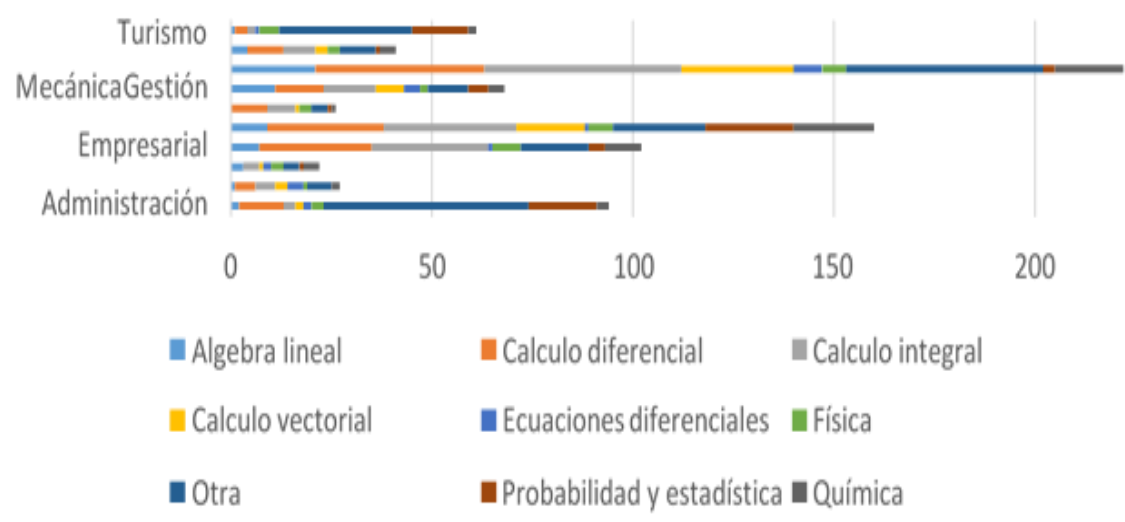

Fuente: Elaboración propia

La motivación se considera como un elemento clave en el involucramiento del individuo que aprende: cuando un alumno está motivado, todo su esfuerzo y personalidad se orienta hacia el logro de una determinada meta, empleando para ello todos sus recursos (Lozano, 2003), dicha motivación nace a partir de las diferentes interacciones con el contexto y agentes sociales y resulta de gran importancia el trato de aceptación o rechazo recibido de los demás, especialmente de los otros significativos, y conforme la base del futuro éxito o fracaso escolar (Sánchez, 2000, en Lozano, 2003) (Gráfica 28).

Gráfica 28. Materias reprobadas por tiempo de estudio.

\section{Materias reprobadas por tiempo de estudio (motivación)}

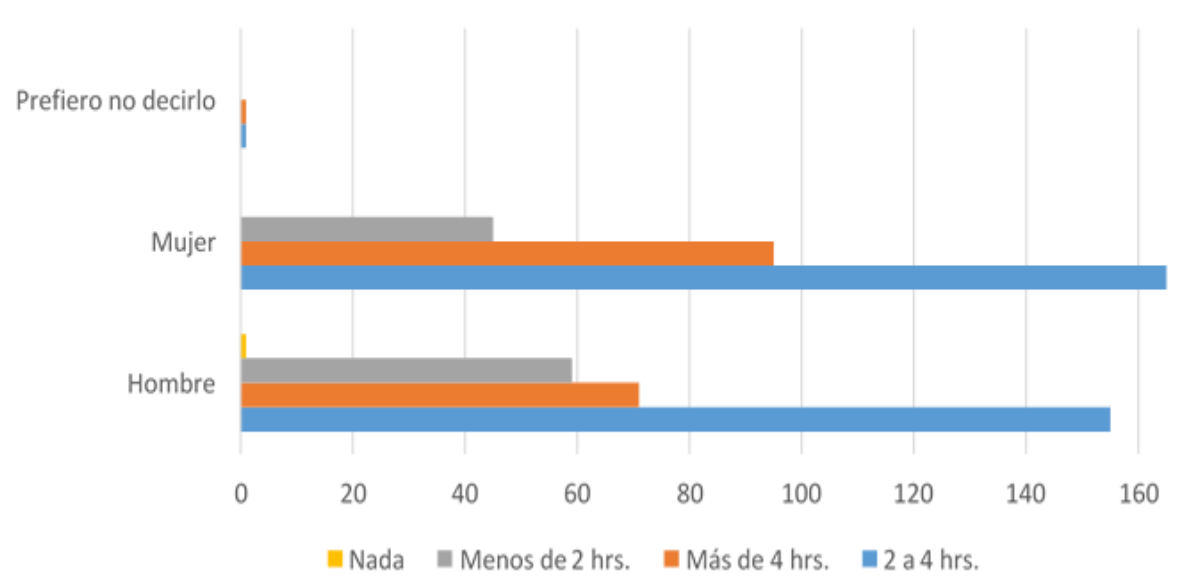

Fuente: Elaboración propia

Ahora bien, respecto a la tercera pregunta subordinada, ¿Cuáles son los factores de origen institucional de mayor incidencia en el índice de reprobación en Ciencias Básicas en los estudiantes del Instituto Tecnológico de San Luis Potosí?, se establece que los factores 
institucionales pueden definirse como "características estructurales y funcionales que difieren en cada institución, y su grado de influencia confiere a la Universidad peculiaridades propias" (Latiesa, 1992 en Montero 2007, p. 219).

En este rubro se encontró que influye de manera directa la manera en la que los docentes preparan y planean sus clases (Gráfica 29), así como el ambiente institucional respecto al proceso de enseñanza-aprendizaje percibido por los estudiantes (Gráfica 30).

Gráfica 29. Relación de materias reprobadas por profesores que preparan clase.

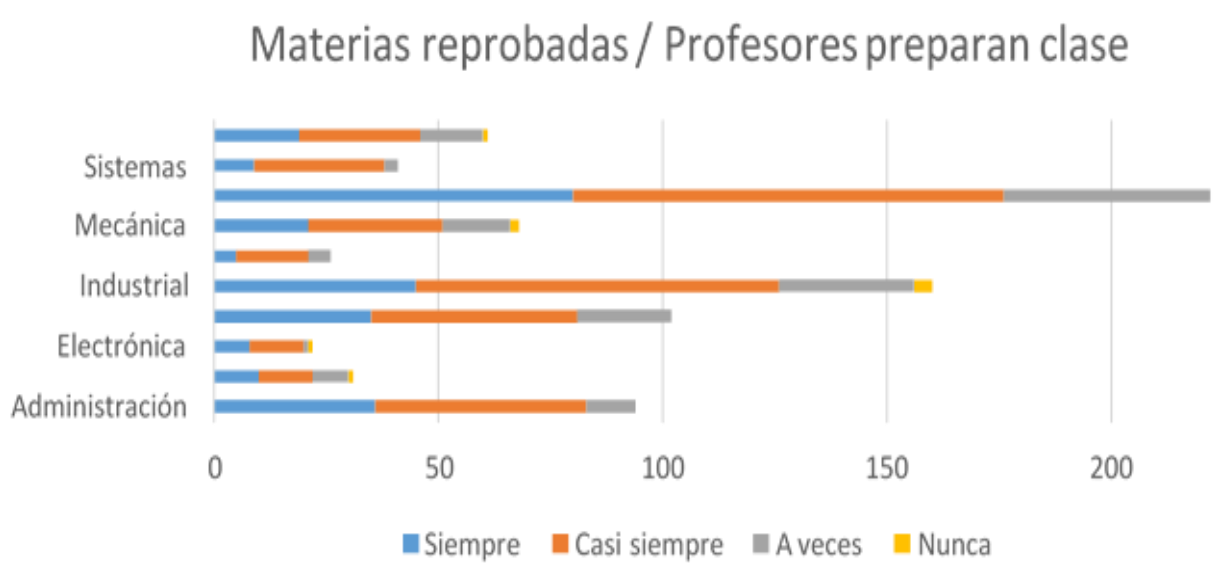

Fuente: Elaboración propia

Gráfica 30. Ambiente institucional respecto al proceso de enseñanza-aprendizaje percibido por los estudiantes

\section{Opinión de los estudiantes respecto a materias reprobadas.}

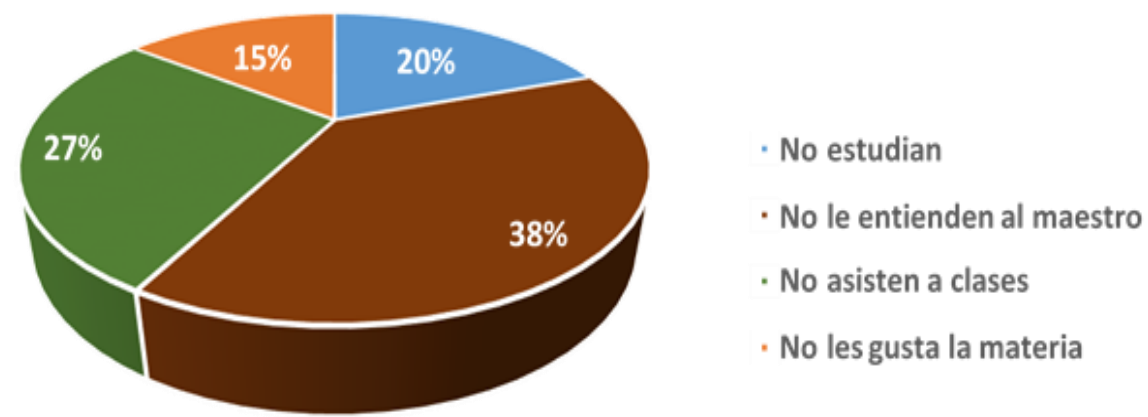

. Fuente: Elaboración propia

Finalmente, la cuarta pregunta subordinada, ¿Cuáles son los factores de mayor incidencia en el índice de reprobación de acuerdo a la percepción de los estudiantes de Ciencias Básicas del Instituto Tecnológico de San Luis Potosí? Refiere al término de autoconcepto académico, el cual influye directamente en el rendimiento global del alumno, de acuerdo 
a Núñez et al., 1998 en Lozano (2003) cuanto mayor es el autoconcepto del alumno, más estrategias de aprendizaje utiliza, lo cual le facilita el procesamiento de la información e influye directamente en el rendimiento a través de su influencia en la motivación intrínseca (Gráfica 31).

Gráfica 31. Percepción de los estudiantes respecto a los altos índices de reprobación.

\section{¿A QUE LE ATRIBUYES QUE EXISTAN ALTOS ÍNDICES DE REPROBACIÓN DEN LAS MATERIAS DE CIENCIAS BÁSICAS}

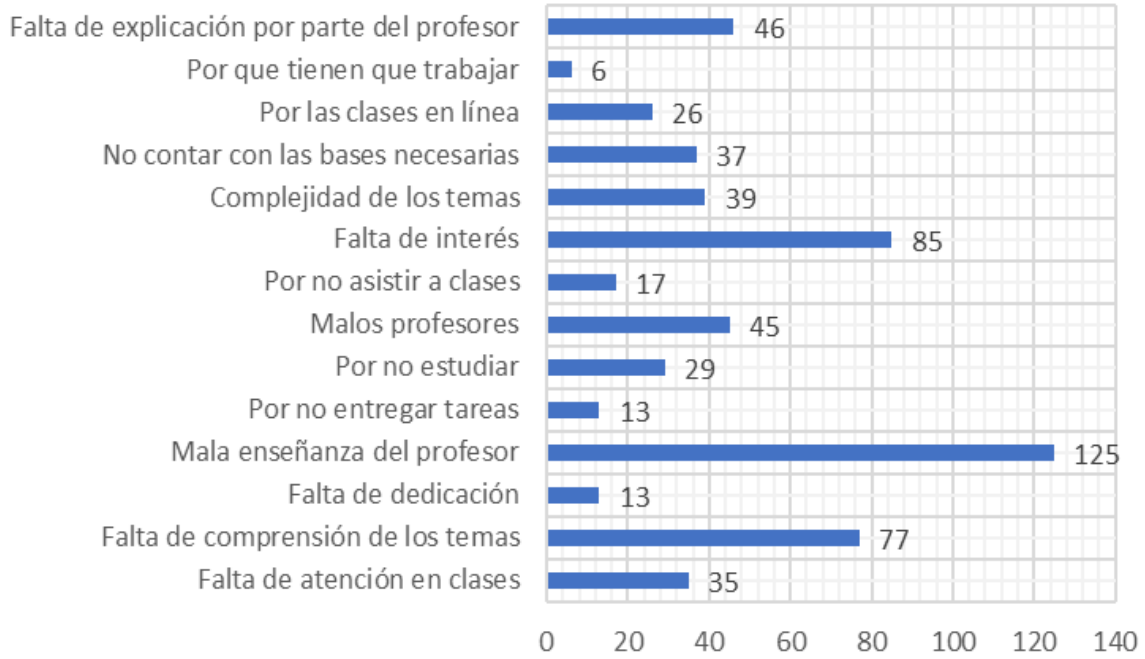

Fuente: Elaboración propia

\section{CONCLUSIÓN O CONSIDERACIONES FINALES}

Las materias del Departamento de Ciencias Básicas conforman el tronco común para las carreras impartidas en el Instituto Tecnológico de San Luis Potosí, habitualmente representan un filtro de permanencia, ya que los estudiantes no cuentan con los conocimientos mínimos necesarios para cursar una carrera universitaria, por lo tanto, estas asignaturas representan una mayor complejidad al inicio de sus estudios.

De los resultados obtenidos se infiere que el índice de reprobación está estrechamente relacionado a los diferentes factores tanto sociales, personales e institucionales. En primer término, dentro de los factores sociales, se pudo observar que situaciones como el trabajar y estudiar al mismo tiempo y un nivel de ingresos familiares bajo afectan directamente el rendimiento escolar. Se percibe que los estudiantes que trabajan no cuentan con el tiempo suficiente para cumplir con sus compromisos escolares, ni para prepararse debidamente para sus clases y/o exámenes, ya que, por lo general, se encuentran agotados, exhaustos y sin ánimos o motivación requeridas para este fin. 
En segundo lugar, el factor personal resultó ser un elemento clave en el logro de su desempeño académico, siendo insuficiente el tiempo que dedican a estudiar, tanto para exámenes como para realizar las diferentes actividades escolares, pues se requiere de mucho más esfuerzo y dedicación el atender una carrera profesional, situación que parece ser ignorada o desconocida por parte de los estudiantes.

Finalmente, en el factor institucional, los estudiantes consideran que la preparación de clases y actividades didácticas que el docente planea para impartir sus clases no es la adecuada, pues a su criterio, no es la ideal para llegar a una comprensión adecuada de los temas, lo que desencadena frustración y desmotivación en los estudiantes, y recae en una falta de interés por atender la materia.

Se sugieren estrategias, tales como formar grupos de apoyo para asesorías por parte de los docentes del área de Ciencias Básicas por materia y dar seguimiento a los estudiantes para coadyuvar a bajar el alto índice de reprobación en dicha área, lo que conlleva a tomar acciones por los directivos, docentes y tutores con propuestas de estrategias para motivar al estudiante a que tome conciencia y le otorguen la importancia a sus estudios y lleguen a concretar una formación educativa para el cumplimiento de sus metas como profesionistas.

\section{LISTA DE REFERENCIAS}

Ayala, S. \&. (2020). Herramientas digitales y reconversión de actividades en los hogares mexicanos. Vicisitudes de la escuela en casa en tiempos de COVID 19. En G. N. Palos, Efectos sociales, económicos, emocionales y de la salud ocasionados por la pandemia del COVID-19: Impactos en Instituciones de Educación Superior y en el Proceso de Enseñanza Aprendizaje (Vol. 1, págs. 83-112). San Luis Potosí, San Luis Potosí, México: Plaza y Valdés Editores. Recuperado el 26 de septiembre de 2021, de https://www.researchgate.net/profile/MarioVenegas/publication/349413322_Estudio_exploratorio_sobre_educacion_remota _durante_COVID-

19_en_Universidad_Tecnologica/links/604c4258458515e529a40589/Estudioexploratorio-sobre-educacion-remota-durante-COVID-19-en

Chavarría, M. (20 de agosto de 2020). Colegio Castillo de Chapultepec. Recuperado el 22 de septiembre de 2021, de SE PUEDE LOGRAR UN APRENDIZAJE 100\% SIGNIFICATIVO CON LA EDUCACIÓN A DISTANCIA: 
https://www.ccch.edu.mx/se-puede-lograr-un-aprendizaje-100-significativo-conla-educacion-a-distancia/

Cívico, A. C. (10. de mayo de 2021). Jóvenes y uso problemático de las tecnologías durante la pandemia: una preocupación familiar. Hachetetepé. Revista científica de educación $\quad y \quad$ comunicación(22), 1-12. doi:https://doi.org/10.25267/Hachetetepe.2021.i22.1204

Delgado, P. (6 de abril de 2020). Observatorio de Innovación Educativa del Tecnológico de Monterrey. (T. d. Monterrey, Editor) Recuperado el 22 de septiembre de 2021, de Padres, alumnos y docentes enfrentan los retos de adaptarse a la educación en línea: https://observatorio.tec.mx/edu-news/educacion-online-retos-escuela-encasa

Estrada, M. (17 de septiembre de 2021). Universidad del Bosque. Recuperado el 30 de septiembre de 2021, de Impacto de las clases virtuales en los procesos de enseñanza y aprendizaje: https://www.unbosque.edu.co/centroinformacion/noticias/impacto-de-las-clases-virtuales-en-los-procesos-deensenanza-y

Fernández, M. H. (8 de julio de 2020). Nexos. Recuperado el 21 de septiembre de 2021, de Jóvenes con un futuro sombrío: media superior ante la pandemia: https://educacion.nexos.com.mx/jovenes-con-un-futuro-sombrio-mediasuperior-ante-la-pandemia/

Flores, G., Torre, L. (1o. de abril de 2020). Universitarios ahogados en tareas online. El Sol de Tampico. Recuperado el 25 de septiembre de 2021, de https://www.elsoldetampico.com.mx/local/regional/adelantan-pago-de-mas-de17-mil-becas-5046587.html

García-Allen, J. (2021). Psicología y Mente. (B. Regader, Editor) Recuperado el 25 de septiembre de 2021, de Los doce estilos de aprendizaje: ¿en qué se basa cada uno?: https://psicologiaymente.com/desarrollo/estilos-de-aprendizaje

Gobernación, S. d. (Ed.). (16 de marzo de 2020). Diario Oficial de la Federación. (Red de publicaciones oficiales mexicanas) Recuperado el 20 de septiembre de 2021, de

https://www.dof.gob.mx/nota_detalle.php?codigo=5589479\&fecha=16/03/2020

GRUPO BANCO MUNDIAL. Educación. (mayo de 2020). Recuperado el 27 de 
septiembre de 2021, de COVID-19: IMPACTO EN LA EDUCACIÓN Y RESPUESTAS DE POLÍTICA PÚBLICA:

https://openknowledge.worldbank.org/bitstream/handle/10986/33696/148198SP. pdf? sequence $=6 \&$ is Allowed $=\mathrm{y}$

Guijosa, C. (26 de febrero de 2019). Obervatorio de Innovación Educativa del Tecnmológico de Monterrey. (T. d. Monterrey, Editor) Recuperado el 21 de septiembre de 2021, de La distracción, un freno para la educación en línea: https://observatorio.tec.mx/edu-news/la-distraccion-un-freno-en-la-educaciononline

Hernández-Sampieri R., F. P. (2018). Metodología de la Investigación. Las rutas cuantitativas, cualitativa y mixta. (1a. ed.). México: Mc Graw Hill.

Lepp, A. B. (13 de enero de 2019). College Students' Multitasking Behavior in Online Versus Face-to-Face Courses. (K. S. University, Ed.) SAGE OPEN, enero-marzo 2019, 1-9. doi:https://doi.org/10.1177/2158244018824505

Liberman, J. L.-B. (27 de abril de 2020). Education for Global Development. Recuperado el 17 de septiembre de 2021, de ¿Siguen aprendiendo los estudiantes durante el COVID-19? La respuesta podría obtenerse a través de la evaluación formativa: https://blogs.worldbank.org/es/education/siguen-aprendiendo-los-estudiantesdurante-el-covid-19-la-respuesta-podria-obtenerse

LinkiaFP Formación profesional oficial. (9 de marzo de 2021). Recuperado el 23 de septiembre de 2021, de Las 8 grandes ventajas de las clases online: https://linkiafp.es/blog/ventajas-clases-online/

Lovón, M. \&. (30 de septiembre de 2020). Repercusiones de las clases virtuales en los estudiantes universitarios en el contexto de la cuarentena por COVID-19: El caso de la PUCP. Propósitos y Representaciones, 8(SPE3). doi:http://dx.doi.org/10.20511/pyr2020.v8nSPE3.588

Mendoza, L. (2020). Lo que la pandemia nos eñseño sobre la educación a distancia. (U. I. México, Ed.) Revista Latinoamericana de Estudios Educativos, 50(ESPECIAL), 343-352. doi:https://doi.org/10.48102/rlee.2020.50.ESPECIAL.119

MEXTUDIA. (24 de julio de 2020). Recuperado el 28 de septiembre de 2021, de ¿Cómo afectó el Confinamiento a estudiantes y profesores?: https://mextudia.com/como- 
afecto-el-confinamiento-a-estudiantes-y-profesores/

Morin, A. (2021). Child Mind Institute. Recuperado el 25 de septiembre de 2021, de 5 razones por las que los estudiantes no están participando en el aprendizaje a distancia: https://childmind.org/es/articulo/5-razones-por-las-que-losestudiantes-no-estan-participando-en-el-aprendizaje-a-distancia/

Murcia, M. (20 de enero de 2021). Los papás de Gabriel \& Adrián. Recuperado el 25 de septiembre de 2021, de ¿Cómo identificar los estilos de aprendizaje en niños?: https://gabrielyadrian.com/tipos-estilos-aprendizaje-ninos/

Navarrete, Z. M. (2020). Políticas implementadas por el gobierno mexicano frente al COVID-19. El caso de la educación básica. (U. Iberoamericana, Ed.) Revista Latinoamericana de Estudios Educativos, L(Especial), 143-172. doi:https://doi.org/10.48102/rlee.2020.50.ESPECIAL.100

Puebla, U. d. (2021). Ventajas de estudiar en línea. Recuperado el 24 de septiembre de 2021, de https://blog.uvp.mx/ventajas-de-estudiar-en-linea/

Ríos, V. H. (2020). Dispositivos, herramientas digitales y plataformas más utilizadas por universitarios mexicanos durante el primer trimestre del confinamiento por el COVID-19. En G. N. Palos, Efectos sociales, económicos, emocionales y de la salud ocasionados por la pandemia del COVID-19: Impactos en Instituciones de Educación Superior y en el Proceso de Enseñanza Aprendizaje (Vol. 1, págs. 355 - 376). San Luis Potosí, San Luis Potosí, México: Plaza y Valdés Editores. Recuperado el 21 de septiembre de 2021, de https://www.researchgate.net/profile/Mario-

Venegas/publication/349413322_Estudio_exploratorio_sobre_educacion_remota _durante_COVID-

19_en_Universidad_Tecnologica/links/604c4258458515e529a40589/Estudioexploratorio-sobre-educacion-remota-durante-COVID-19-en

Romero, L., Salinas, V. \& Mortera, F. (abril de 2010). Estilos de aprendizaje basados en el modelo de Kolb en la educación virtual. (G. M. Dávila, Ed.) Apertura, 2(1), 7285. Recuperado el 24 de septiembre de 2021, de http://www.udgvirtual.udg.mx/apertura/index.php/apertura/article/view/21/30

Zavala, M. (4 de diciembre de 2020). Se disparan quejas por mal servicio de Internet. Incrementaron 100 por ciento los reportes de fallas en pandemia. El Heraldo de 
México. Recuperado el 29 de septiembre de 2021, de https://heraldodemexico.com.mx/nacional/2020/12/4/se-disparan-quejas-pormal-servicio-de-internet-232161.html 